Network Working Group

Request for Comments: 5101

Category: Standards Track
B. Claise, Ed. Cisco Systems, Inc. January 2008

\title{
Specification of the IP Flow Information Export (IPFIX) Protocol for the Exchange of IP Traffic Flow Information
}

Status of This Memo

This document specifies an Internet standards track protocol for the Internet community, and requests discussion and suggestions for improvements. Please refer to the current edition of the "Internet Official Protocol standards" (STD 1) for the standardization state and status of this protocol. Distribution of this memo is unlimited.

Abstract

This document specifies the IP Flow Information Export (IPFIX) protocol that serves for transmitting IP Traffic Flow information over the network. In order to transmit IP Traffic Flow information from an Exporting Process to an information Collecting Process, a common representation of flow data and a standard means of communicating them is required. This document describes how the IPFIX Data and Template Records are carried over a number of transport protocols from an IPFIX Exporting Process to an IPFIX Collecting Process.

Table of Contents

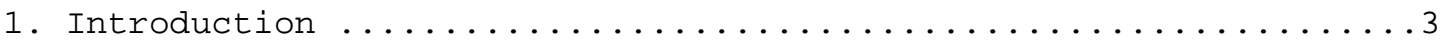

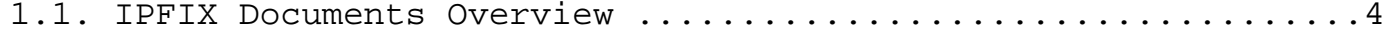

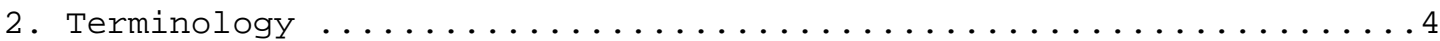

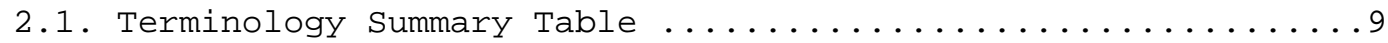

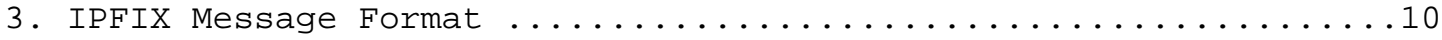

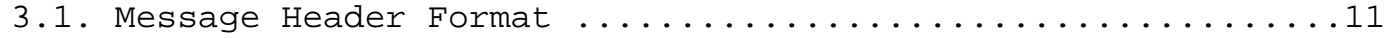

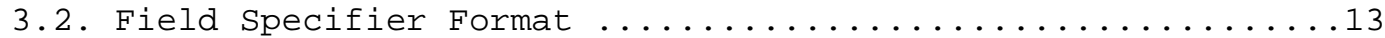

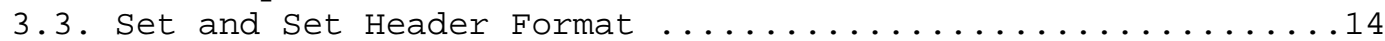

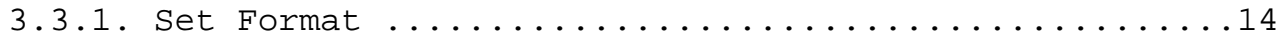

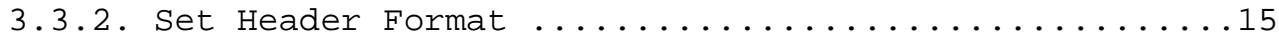

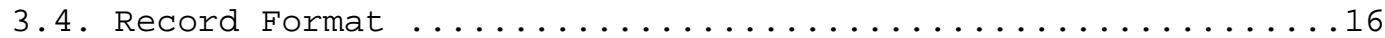

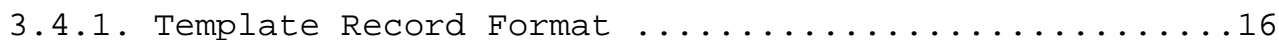

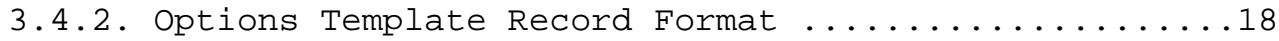

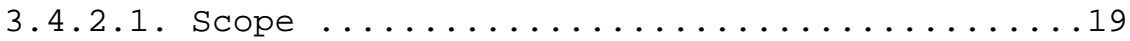

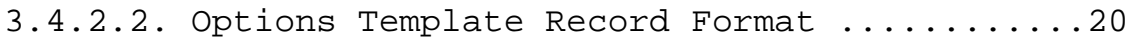

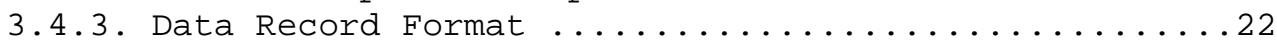

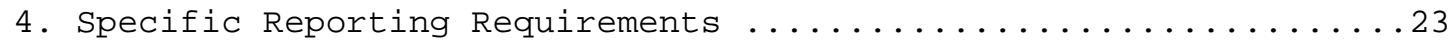

4.1. The Metering Process Statistics Option Template .......23 
4.2. The Metering Process Reliability Statistics Option

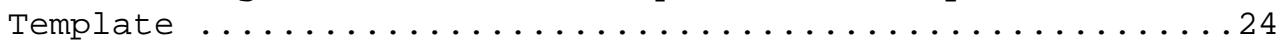

4.3. The Exporting Process Reliability Statistics

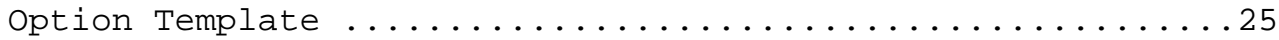

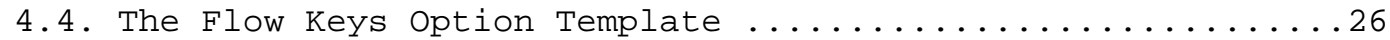

5. IPFIX Message Header "Export Time" and Flow Record Time ......27

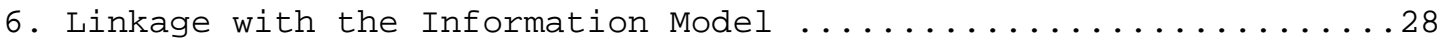

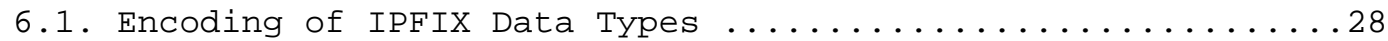

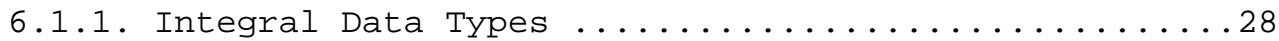

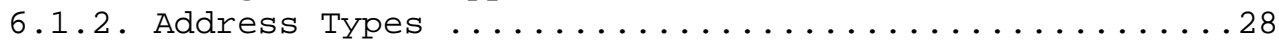

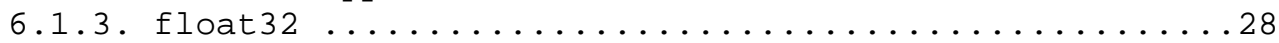

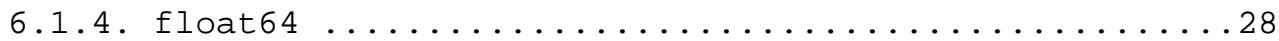

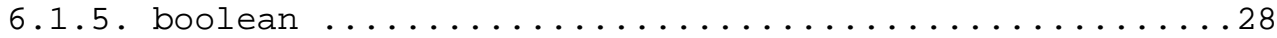

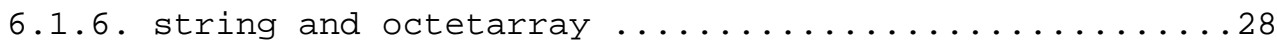

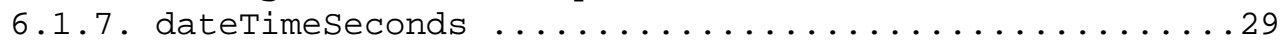

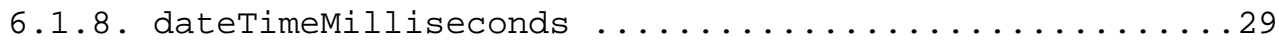

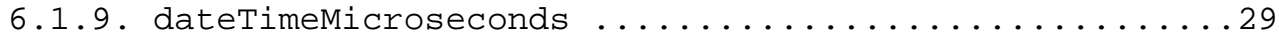

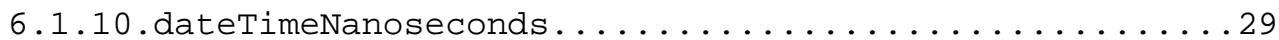

6.2. Reduced Size Encoding of Integer and Float Types .......29

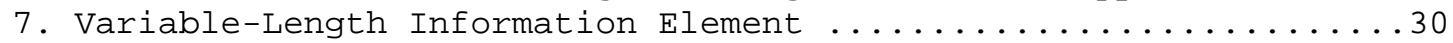

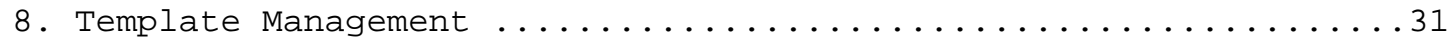

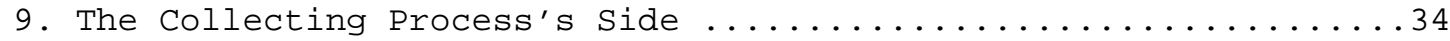

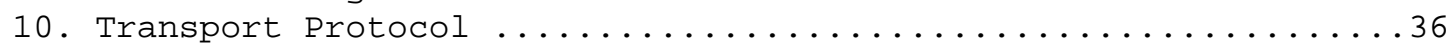

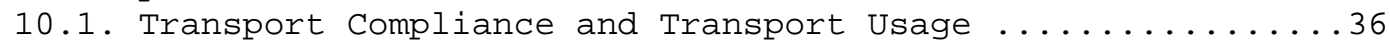

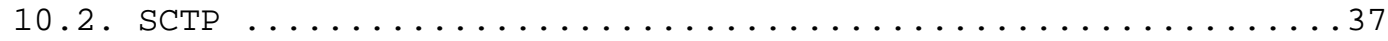

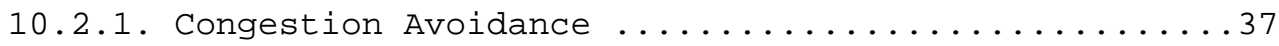

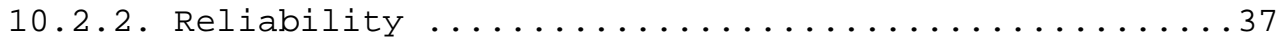

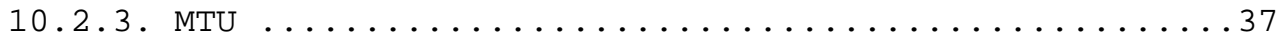

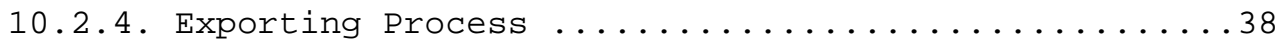

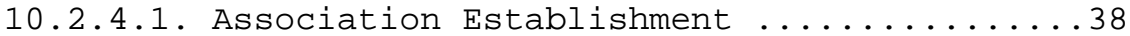

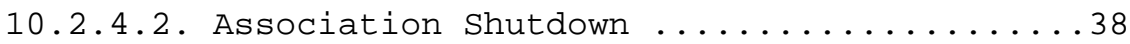

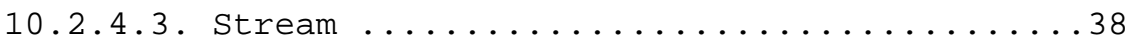

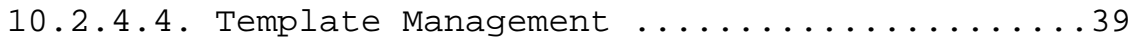

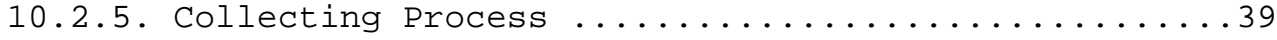

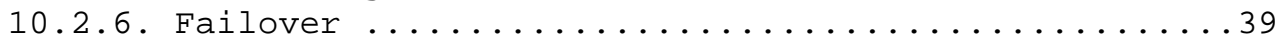

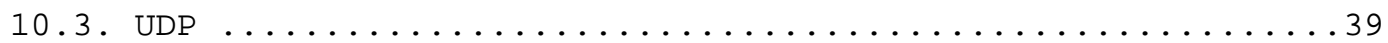

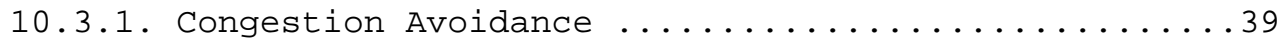

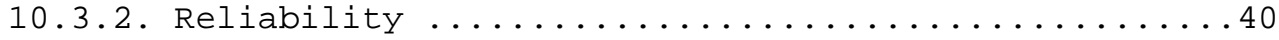

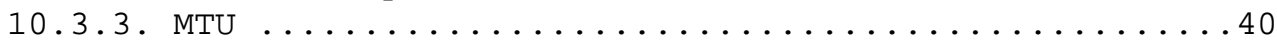

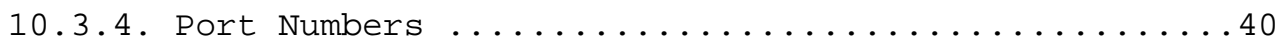

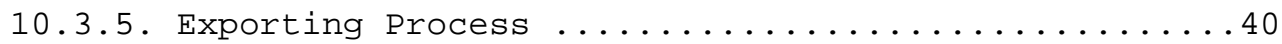

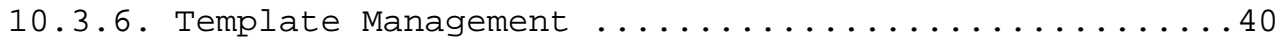

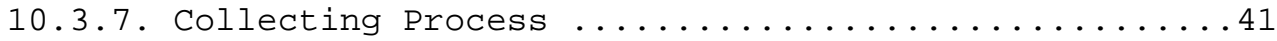

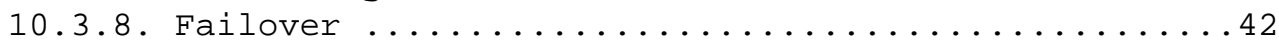

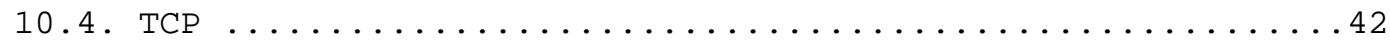

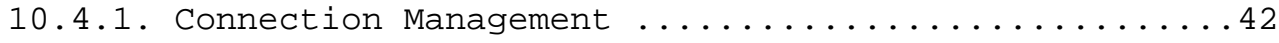

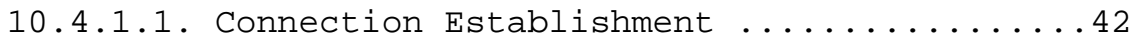

10.4.1.2. Graceful Connection Release ..........43 
10.4.1.3. Restarting Interrupted Connections .....43

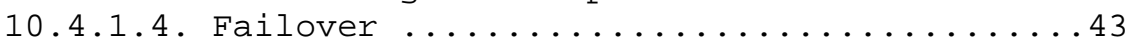

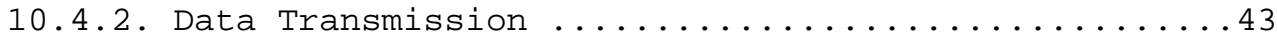

10.4.2.1. IPFIX Message Encoding ..............43

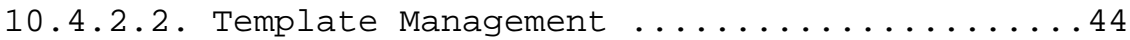

10.4.2.3. Congestion Handling and Reliability .....44

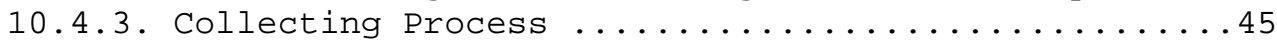

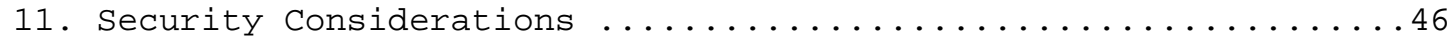

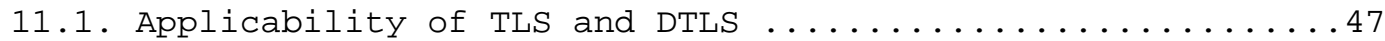

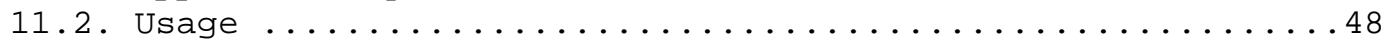

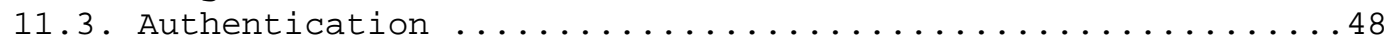

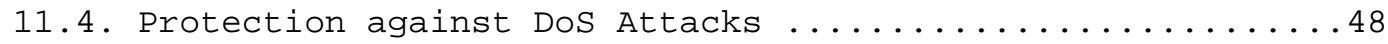

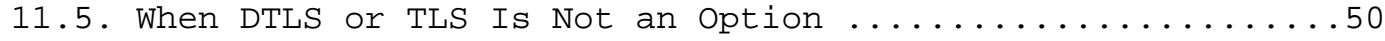

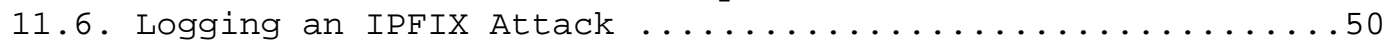

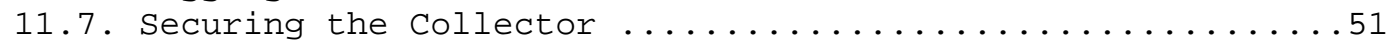

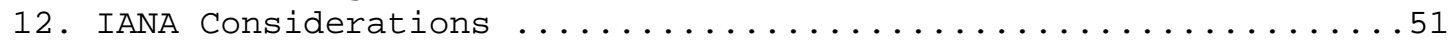

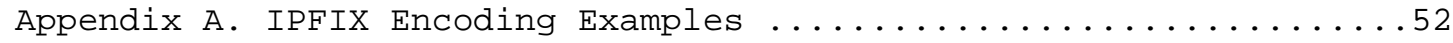

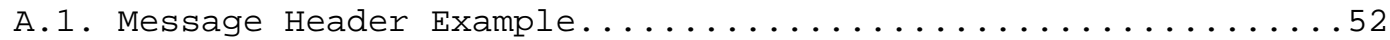

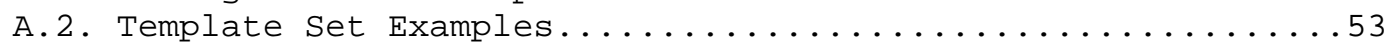

A.2.1. Template Set Using IETF-Specified Information

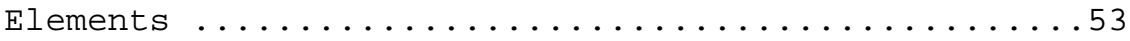

A.2.2. Template Set Using Enterprise-Specific Information

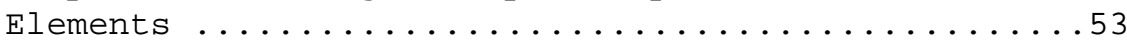

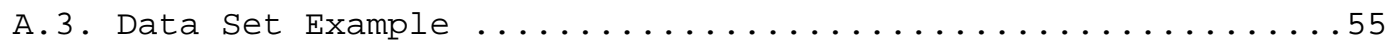

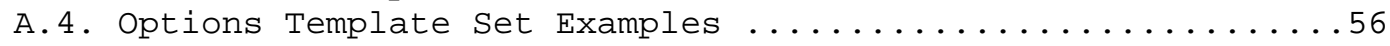

A.4.1. Options Template Set Using IETF-Specified

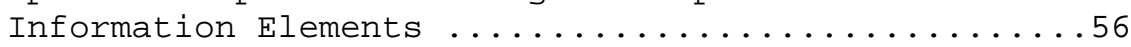

A.4.2. Options Template Set Using Enterprise-Specific

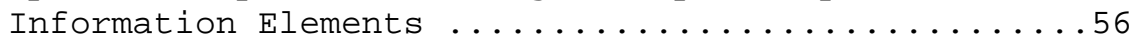

A.4.3. Options Template Set Using an Enterprise-Specific

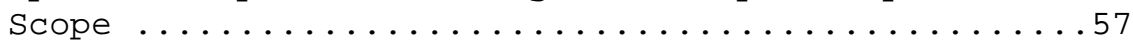

A.4.4. Data Set Using an Enterprise-Specific Scope .....58

A.5. Variable-Length Information Element Examples .........59

A.5.1. Example of Variable-Length Information Element

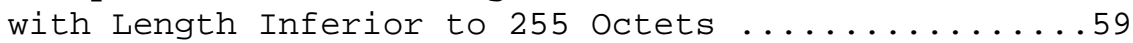

A.5.2. Example of Variable-Length Information Element

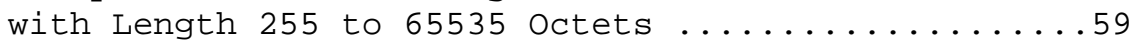

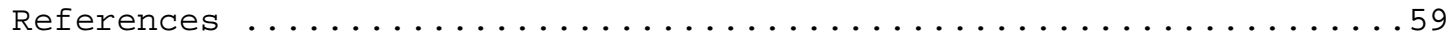

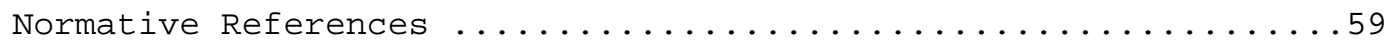

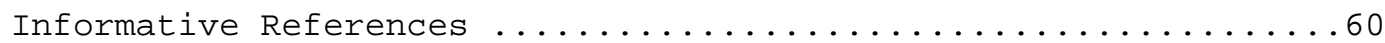

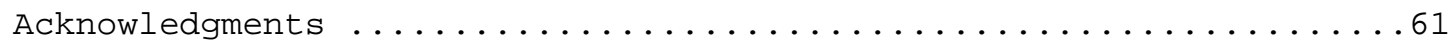

\section{Introduction}

A data network with IP traffic primarily consists of IP flows passing through the network elements. It is often interesting, useful, or even required to have access to information about these flows that pass through the network elements for administrative or other 
purposes. The IPFIX Collecting Process should be able to receive the flow information passing through multiple network elements within the data network. This requires uniformity in the method of representing the flow information and the means of communicating the flows from the network elements to the collection point. This document specifies the protocol to achieve these aforementioned requirements. This document specifies in detail the representation of different flows, the additional data required for flow interpretation, packet format, transport mechanisms used, security concerns, etc.

\subsection{IPFIX Documents Overview}

The IPFIX protocol provides network administrators with access to IP flow information. The architecture for the export of measured IP flow information out of an IPFIX Exporting Process to a Collecting Process is defined in [IPFIX-ARCH], per the requirements defined in [RFC3917]. This document specifies how IPFIX data records and templates are carried via a number of transport protocols from IPFIX Exporting Processes to IPFIX Collecting Processes. IPFIX has a formal description of IPFIX Information Elements, their name, type and additional semantic information, as specified in [RFC5102]. Finally, [IPFIX-AS] describes what type of applications can use the IPFIX protocol and how they can use the information provided. It furthermore shows how the IPFIX framework relates to other architectures and frameworks.

2. Terminology

The key words "MUST", "MUST NOT", "REQUIRED", "SHALL", "SHALL NOT", "SHOULD", "SHOULD NOT", "RECOMMENDED", "MAY", and "OPTIONAL" in this document are to be interpreted as described in RFC 2119 [RFC2119].

The definitions of the basic terms like IP Traffic Flow, Exporting Process, Collecting Process, Observation Points, etc. are semantically identical to those found in the IPFIX requirements document [RFC3917]. Some of the terms have been expanded for more clarity when defining the protocol. Additional terms required for the protocol have also been defined. Definitions in this document and in [IPFIX-ARCH] are equivalent, except that definitions that are only relevant to the IPFIX protocol only appear here.

The terminology summary table in section 2.1 gives a quick overview of the relationships between some of the different terms defined. 
Observation Point

An Observation Point is a location in the network where IP packets can be observed. Examples include: a line to which a probe is attached, a shared medium, such as an Ethernet-based LAN, a single port of a router, or a set of interfaces (physical or logical) of a router.

Note that every Observation Point is associated with an Observation Domain (defined below), and that one observation Point may be a superset of several other observation Points. For example, one Observation Point can be an entire line card. That would be the superset of the individual Observation points at the line card's interfaces.

Observation Domain

An Observation Domain is the largest set of Observation Points for which Flow information can be aggregated by a Metering Process. For example, a router line card may be an Observation Domain if it is composed of several interfaces, each of which is an observation Point. In the IPFIX Message it generates, the Observation Domain includes its Observation Domain ID, which is unique per Exporting Process. That way, the Collecting Process can identify the specific Observation Domain from the Exporter that sends the IPFIX Messages. Every Observation Point is associated with an Observation Domain. It is RECOMMENDED that Observation Domain IDs also be unique per IPFIX Device.

IP Traffic Flow or Flow

There are several definitions of the term 'flow' being used by the Internet community. Within the context of IPFIX we use the following definition:

A Flow is defined as a set of IP packets passing an observation Point in the network during a certain time interval. All packets belonging to a particular flow have a set of common properties. Each property is defined as the result of applying a function to the values of:

1. one or more packet header fields (e.g., destination IP address), transport header fields (e.g., destination port number), or application header fields (e.g., RTP header fields [RFC3550]).

2. one or more characteristics of the packet itself (e.g., number of MPLS labels, etc...). 
3. one or more of fields derived from packet treatment (e.g., next hop IP address, the output interface, etc...).

A packet is defined as belonging to a Flow if it completely satisfies all the defined properties of the Flow.

This definition covers the range from a Flow containing all packets observed at a network interface to a Flow consisting of just a single packet between two applications. It includes packets selected by a sampling mechanism.

Flow Key

Each of the fields that:

1. belong to the packet header (e.g., destination IP address),

2. are a property of the packet itself (e.g., packet length),

3. are derived from packet treatment (e.g., Autonomous System (AS) number),

and that are used to define a Flow are termed Flow Keys.

Flow Record

A Flow Record contains information about a specific Flow that was observed at an Observation Point. A Flow Record contains measured properties of the Flow (e.g., the total number of bytes for all the Flow's packets) and usually characteristic properties of the Flow (e.g., source IP address).

Metering Process

The Metering Process generates Flow Records. Inputs to the process are packet headers and characteristics observed at an Observation Point, and packet treatment at the Observation Point (for example, the selected output interface).

The Metering Process consists of a set of functions that includes packet header capturing, timestamping, sampling, classifying, and maintaining Flow Records.

The maintenance of Flow Records may include creating new records, updating existing ones, computing Flow statistics, deriving further flow properties, detecting Flow expiration, passing Flow Records to the Exporting Process, and deleting Flow Records. 
Exporting Process

The Exporting Process sends Flow Records to one or more Collecting Processes. The Flow Records are generated by one or more Metering Processes.

Exporter

A device that hosts one or more Exporting Processes is termed an Exporter.

IPFIX Device

An IPFIX Device hosts at least one Exporting Process. It may host further Exporting Processes and arbitrary numbers of observation Points and Metering Processes.

Collecting Process

A Collecting Process receives flow Records from one or more Exporting Processes. The Collecting Process might process or store received Flow Records, but such actions are out of scope for this document.

Collector

A device that hosts one or more Collecting Processes is termed a collector.

Template

A Template is an ordered sequence of <type, length> pairs used to completely specify the structure and semantics of a particular set of information that needs to be communicated from an IPFIX Device to a Collector. Each Template is uniquely identifiable by means of a Template ID.

IPFIX Message

An IPFIX Message is a message originating at the Exporting Process that carries the IPFIX records of this Exporting Process and whose destination is a Collecting Process. An IPFIX Message is encapsulated at the transport layer. 
Message Header

The Message Header is the first part of an IPFIX Message, which provides basic information about the message, such as the IPFIX version, length of the message, message sequence number, etc.

Template Record

A Template Record defines the structure and interpretation of fields in a Data Record.

Data Record

A Data Record is a record that contains values of the parameters corresponding to a Template Record.

Options Template Record

An Options Template Record is a Template Record that defines the structure and interpretation of fields in a Data Record, including defining how to scope the applicability of the Data Record.

Set

Set is a generic term for a collection of records that have a similar structure. In an IPFIX Message, one or more sets follow the Message Header.

There are three different types of Sets: Template set, Options Template Set, and Data set.

Template set

A Template set is a collection of one or more Template Records that have been grouped together in an IPFIX Message.

Options Template set

An Options Template Set is a collection of one or more Options Template Records that have been grouped together in an IPFIX Message.

Data set

A Data set is one or more Data Records, of the same type, that are grouped together in an IPFIX Message. Each Data Record is previously defined by a Template Record or an Options Template Record. 


\section{Information Element}

An Information Element is a protocol and encoding-independent description of an attribute that may appear in an IPFIX Record. The IPFIX information model [RFC5102] defines the base set of Information Elements for IPFIX. The type associated with an Information Element indicates constraints on what it may contain and also determines the valid encoding mechanisms for use in IPFIX.

Transport Session

In Stream Control Transmission Protocol (SCTP), the transport session is known as the SCTP association, which is uniquely identified by the SCTP endpoints [RFC4960]; in TCP, the transport session is known as the TCP connection, which is uniquely identified by the combination of IP addresses and TCP ports used. In UDP, the transport session is known as the UDP session, which is uniquely identified by the combination of IP addresses and UDP ports used.

\subsection{Terminology Summary Table}

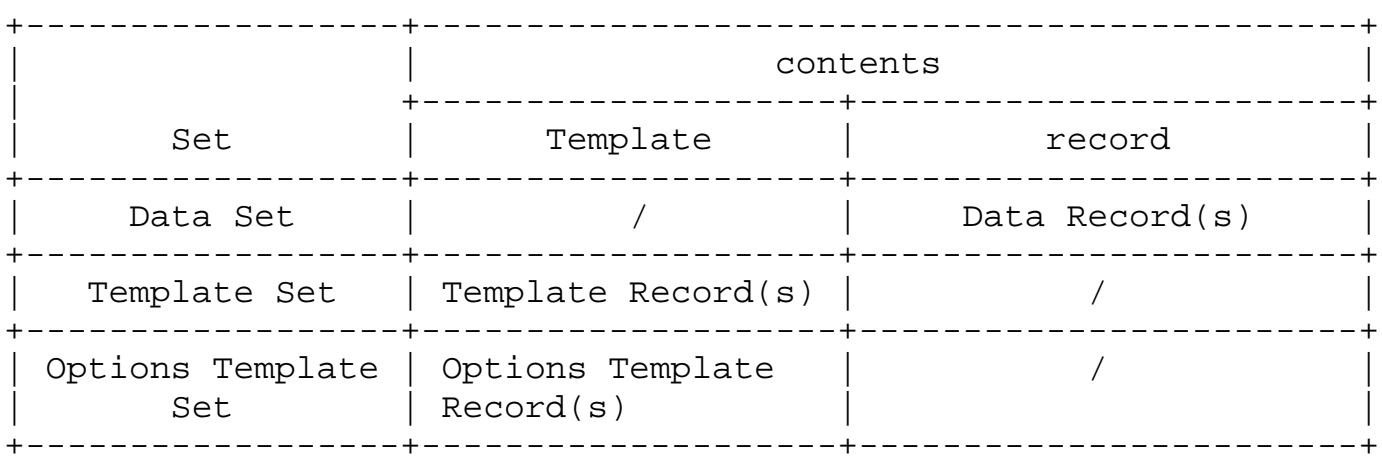

Figure A: Terminology Summary Table

A Data Set is composed of Data Record(s). No Template Record is included. A Template Record or an Options Template Record defines the Data Record.

A Template set contains only Template Record(s).

An Options Template Set contains only Options Template Record(s). 


\section{IPFIX Message Format}

An IPFIX Message consists of a Message Header, followed by one or more sets. The sets can be any of the possible three types: Data Set, Template set, or Options Template set.

The format of the IPFIX Message is shown in Figure $B$.

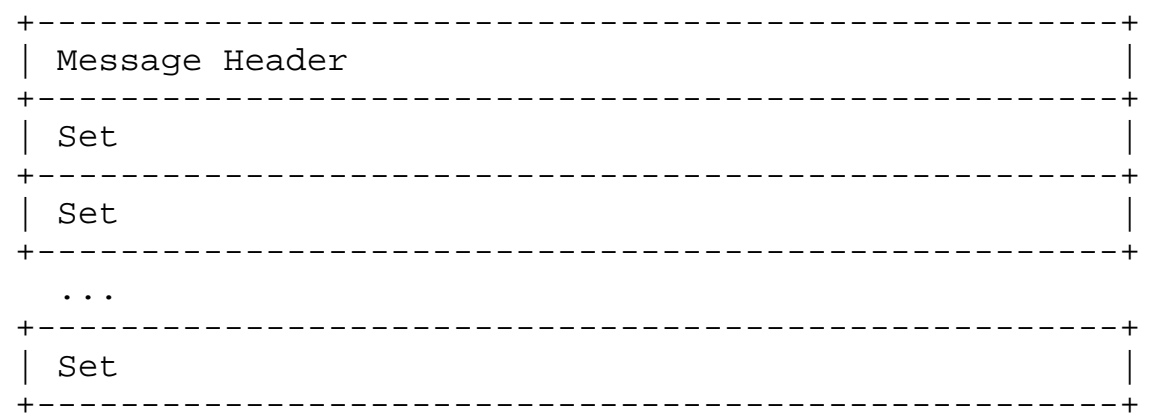

Figure B: IPFIX Message Format

The Exporter MUST code all binary integers of the Message Header and the different sets in network-byte order (also known as the big-endian byte ordering).

Following are some examples of IPFIX Messages:

1. An IPFIX Message consisting of interleaved Template, Data, and Options Template Sets -- A newly created Template is exported as soon as possible. So, if there is already an IPFIX Message with a Data set that is being prepared for export, the Template and Option Template sets are interleaved with this information, subject to availability of space.

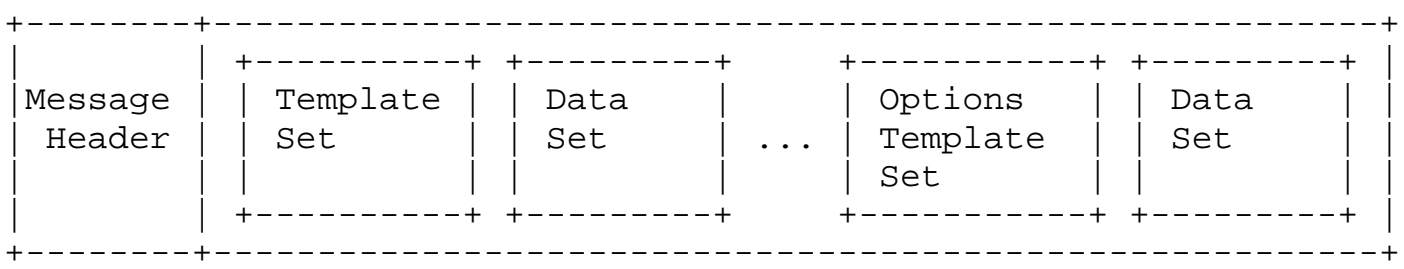

Figure C: IPFIX Message, Example 1 
2. An IPFIX Message consisting entirely of Data Sets -- After the appropriate Template Records have been defined and transmitted to the Collecting Process, the majority of IPFIX Messages consist solely of Data Sets.

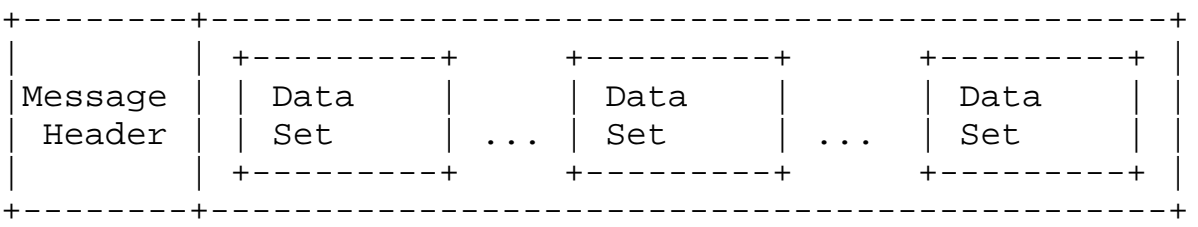

Figure D: IPFIX Message, Example 2

3. An IPfIX Message consisting entirely of Template and Options Template Sets.

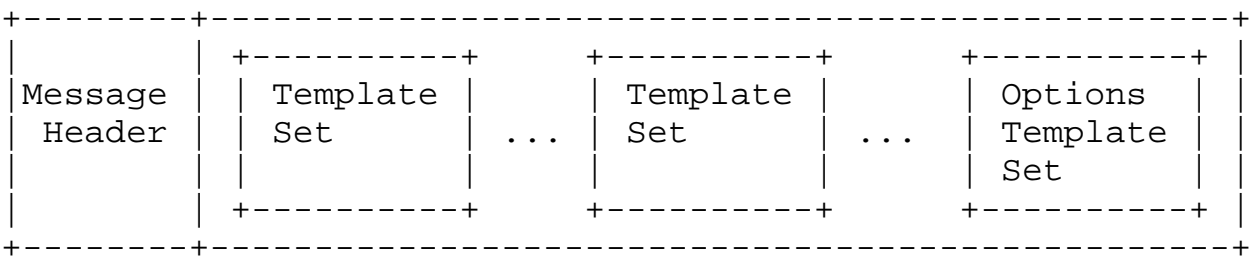

Figure E: IPFIX Message, Example 3

3.1. Message Header Format

The format of the IPFIX Message Header is shown in Figure F.

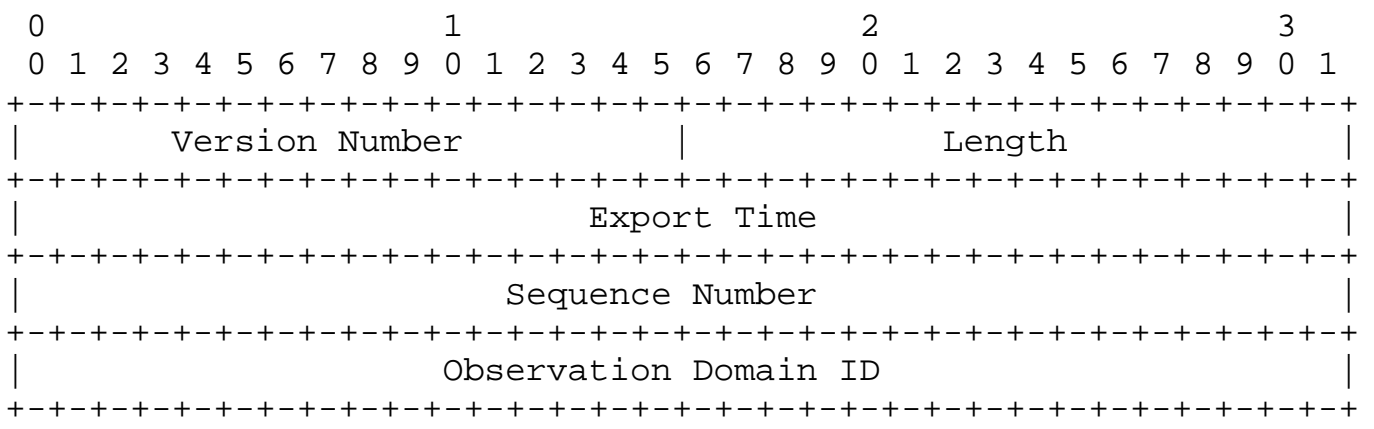

Figure F: IPFIX Message Header Format 
Message Header Field Descriptions:

Version

Version of Flow Record format exported in this message. The value of this field is 0x000a for the current version, incrementing by one the version used in the NetFlow services export version 9 [RFC3954].

Length

Total length of the IPFIX Message, measured in octets, including Message Header and Set(s).

Export Time

Time, in seconds, since 0000 UTC Jan 1, 1970, at which the IPFIX Message Header leaves the Exporter.

Sequence Number

Incremental sequence counter modulo $2^{\wedge} 32$ of all IPFIX Data Records sent on this PR-SCTP stream from the current Observation Domain by the Exporting Process. Check the specific meaning of this field in the subsections of Section 10 when UDP or TCP is selected as the transport protocol. This value SHOULD be used by the Collecting Process to identify whether any IPFIX Data Records have been missed. Template and Options Template Records do not increase the Sequence Number.

Observation Domain ID

A 32-bit identifier of the Observation Domain that is locally unique to the Exporting Process. The Exporting Process uses the Observation Domain ID to uniquely identify to the collecting Process the Observation Domain that metered the Flows. It is RECOMMENDED that this identifier also be unique per IPFIX Device. Collecting Processes SHOULD use the Transport Session and the Observation Domain ID field to separate different export streams originating from the same Exporting Process. The observation Domain ID SHOULD be 0 when no specific Observation Domain ID is relevant for the entire IPFIX Message, for example, when exporting the Exporting Process Statistics, or in case of a hierarchy of Collectors when aggregated Data Records are exported. 


\subsection{Field Specifier Format}

Vendors need the ability to define proprietary Information Elements, because, for example, they are delivering a pre-standards product, or the Information Element is, in some way, commercially sensitive. This section describes the Field specifier format for both IETF-specified Information Elements [RFC5102] and enterprise-specific Information Elements.

The Information Elements are identified by the Information Element identifier. When the Enterprise bit is set to 0 , the corresponding Information Element identifier will report an IETF-specified Information Element, and the Enterprise Number MUST NOT be present. When the Enterprise bit is set to 1, the corresponding Information Element identifier will report an enterprise-specific Information Element; the Enterprise Number MUST be present. An example of this is shown in Section A.4.2.

The Field Specifier format is shown in Figure G.

$\begin{array}{llllllllllllllll}0 & 1 & 2 & 3\end{array}$

$\begin{array}{llllllllllllllllllllllllllllllll}0 & 1 & 2 & 3 & 4 & 5 & 6 & 7 & 8 & 9 & 0 & 1 & 2 & 3 & 4 & 5 & 6 & 7 & 8 & 9 & 0 & 1 & 2 & 3 & 4 & 5 & 6 & 7 & 8 & 9 & 0 & 1\end{array}$

+-+-+-+-+-+-+-+-+-+-+-+-+-+-+-+-+-+-+-+-+-+-+-+-+-+-+-+-+-+-+-+-t $|\mathrm{E}|$ Information Element ident. Field Length

$+-+-+-+-+-+-+-+-+-+-+-+-+-+-+-+-+-+-+-+-+-+-+-+-+-+-+-+-+-+-+-+-+$

Enterprise Number

Figure G: Field Specifier Format

Where:

$\mathrm{E}$

Enterprise bit. This is the first bit of the Field specifier. If this bit is zero, the Information Element Identifier identifies an IETF-specified Information Element, and the four-octet Enterprise Number field MUST NOT be present. If this bit is one, the Information Element identifier identifies an enterprise-specific Information Element, and the Enterprise Number filed MUST be present.

Information Element identifier

A numeric value that represents the type of Information Element. Refer to [RFC5102]. 
Field Length

The length of the corresponding encoded Information Element, in octets. Refer to [RFC5102]. The field length may be smaller than the definition in [RFC5102] if the reduced size encoding is used (see section 6.2). The value 65535 is reserved for variablelength Information Elements (see Section 7).

Enterprise Number

IANA enterprise number [PEN] of the authority defining the Information Element identifier in this Template Record.

3.3. Set and Set Header Format

A Set is a generic term for a collection of records that have a similar structure. There are three different types of sets: Template Sets, Options Template Sets, and Data Sets. Each of these sets consists of a set Header and one or more records. The Set Format and the set Header Format are defined in the following sections.

\subsubsection{Set Format}

A set has the format shown in Figure $H$. The record types can be either Template Records, Options Template Records, or Data Records. The record types MUST NOT be mixed within a set.

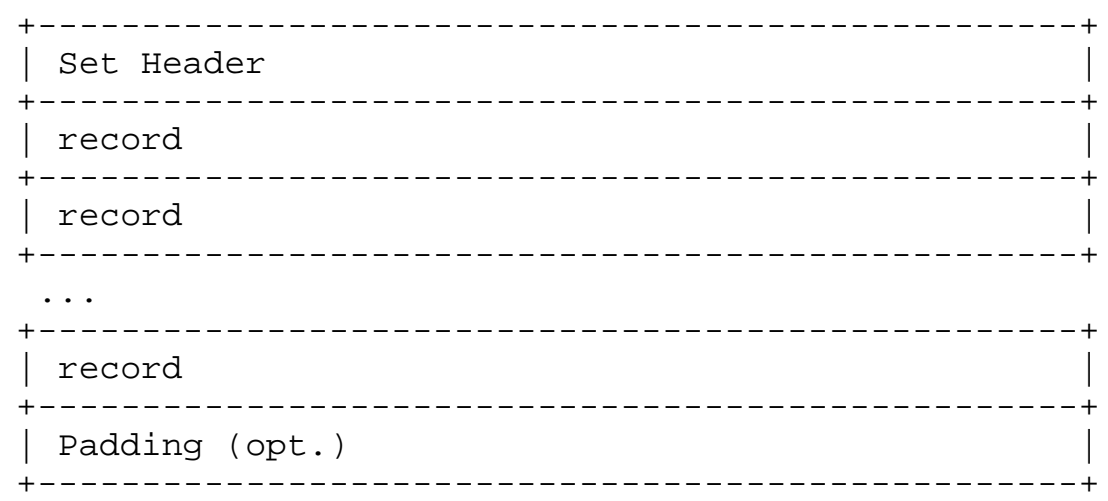

Figure H: Set Format

The Set Field Definitions are as follows:

Set Header

The Set Header Format is defined in Section 3.3.2. 
Record

One of the record Formats: Template Record, Options Template Record, or Data Record Format.

\section{Padding}

The Exporting Process MAY insert some padding octets, so that the subsequent set starts at an aligned boundary. For security reasons, the padding octet(s) MUST be composed of zero (0) valued octets. The padding length MUST be shorter than any allowable record in this set. If padding of the IPFIX Message is desired in combination with very short records, then the padding Information Element 'paddingoctets' [RFC5102] can be used for padding records such that their length is increased to a multiple of 4 or 8 octets. Because Template Sets are always 4-octet aligned by definition, padding is only needed in case of other alignments e.g., on 8-octet boundaries.

\subsubsection{Set Header Format}

Every set contains a common header. This header is defined in Figure I.

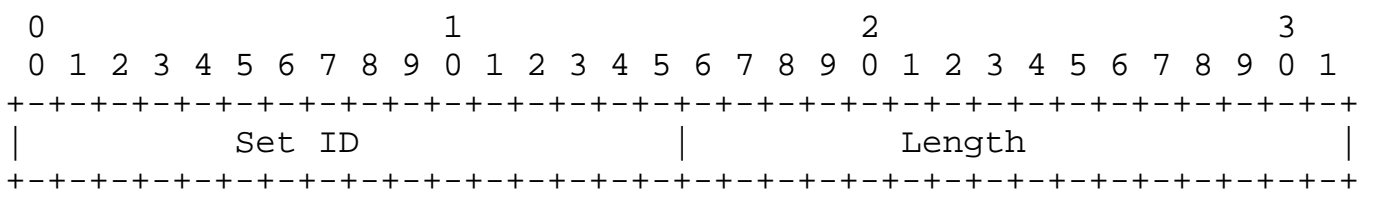

Figure I: Set Header Format

The Set Header Field Definitions are as follows:

Set ID

Set ID value identifies the set. A value of 2 is reserved for the Template Set. A value of 3 is reserved for the Option Template Set. All other values from 4 to 255 are reserved for future use. Values above 255 are used for Data Sets. The Set ID values of 0 and 1 are not used for historical reasons [RFC3954].

Length

Total length of the set, in octets, including the set Header, all records, and the optional padding. Because an individual Set MAY contain multiple records, the Length value MUST be used to determine the position of the next set. 


\subsection{Record Format}

IPFIX defines three record formats, defined in the next sections: the Template Record Format, the Options Template Record Format, and the Data Record Format.

\subsubsection{Template Record Format}

One of the essential elements in the IPFIX record format is the Template Record. Templates greatly enhance the flexibility of the record format because they allow the collecting Process to process IPFIX Messages without necessarily knowing the interpretation of all Data Records. A Template Record contains any combination of IANA-assigned and/or enterprise-specific Information Elements identifiers.

The format of the Template Record is shown in Figure J. It consists of a Template Record Header and one or more Field Specifiers. The definition of the Field Specifiers is given in Figure G above.

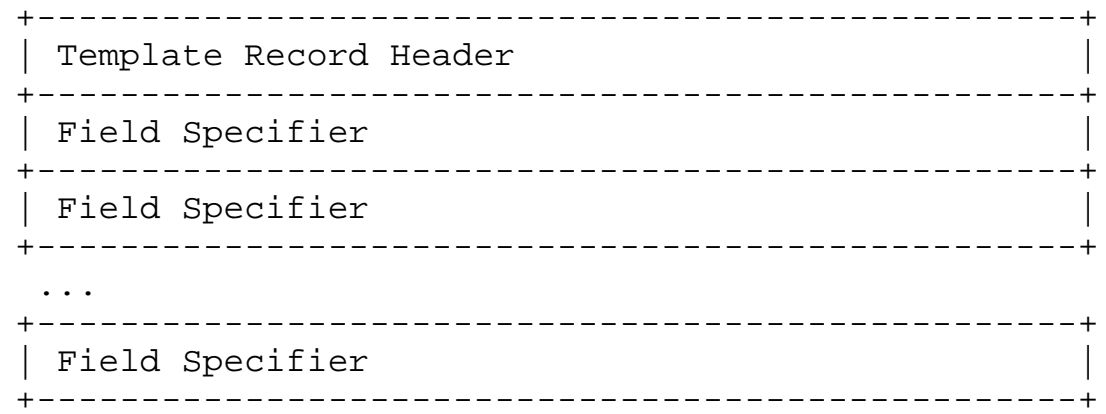

Figure J: Template Record Format

The format of the Template Record Header is shown in Figure $K$. $\begin{array}{llllllllllllll}0 & 1 & 2 & 3\end{array}$

$\begin{array}{lllllllllllllllllllllllllllllllll}0 & 1 & 2 & 3 & 4 & 5 & 6 & 7 & 8 & 9 & 0 & 1 & 2 & 3 & 4 & 5 & 6 & 7 & 8 & 9 & 0 & 1 & 2 & 3 & 4 & 5 & 6 & 7 & 8 & 9 & 0 & 1\end{array}$

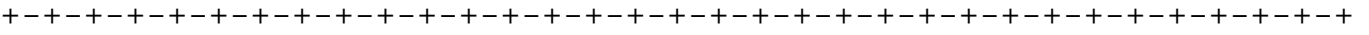
$\mid$\begin{tabular}{c|c} 
Template ID $(>255)$ & Field Count
\end{tabular}

Figure K: Template Record Header Format 
The Template Record Header Field Definitions are as follows:

Template ID

Each of the newly generated Template Records is given a unique Template ID. This uniqueness is local to the Transport session and Observation Domain that generated the Template ID. Template IDs 0-255 are reserved for Template Sets, Options Template Sets, and other reserved Sets yet to be created. Template IDs of Data Sets are numbered from 256 to 65535. There are no constraints regarding the order of the Template ID allocation.

Field Count

Number of fields in this Template Record.

The example in Figure L shows a Template set with mixed standard and enterprise-specific Information Elements. It consists of a set Header, a Template Header, and several Field Specifiers. 


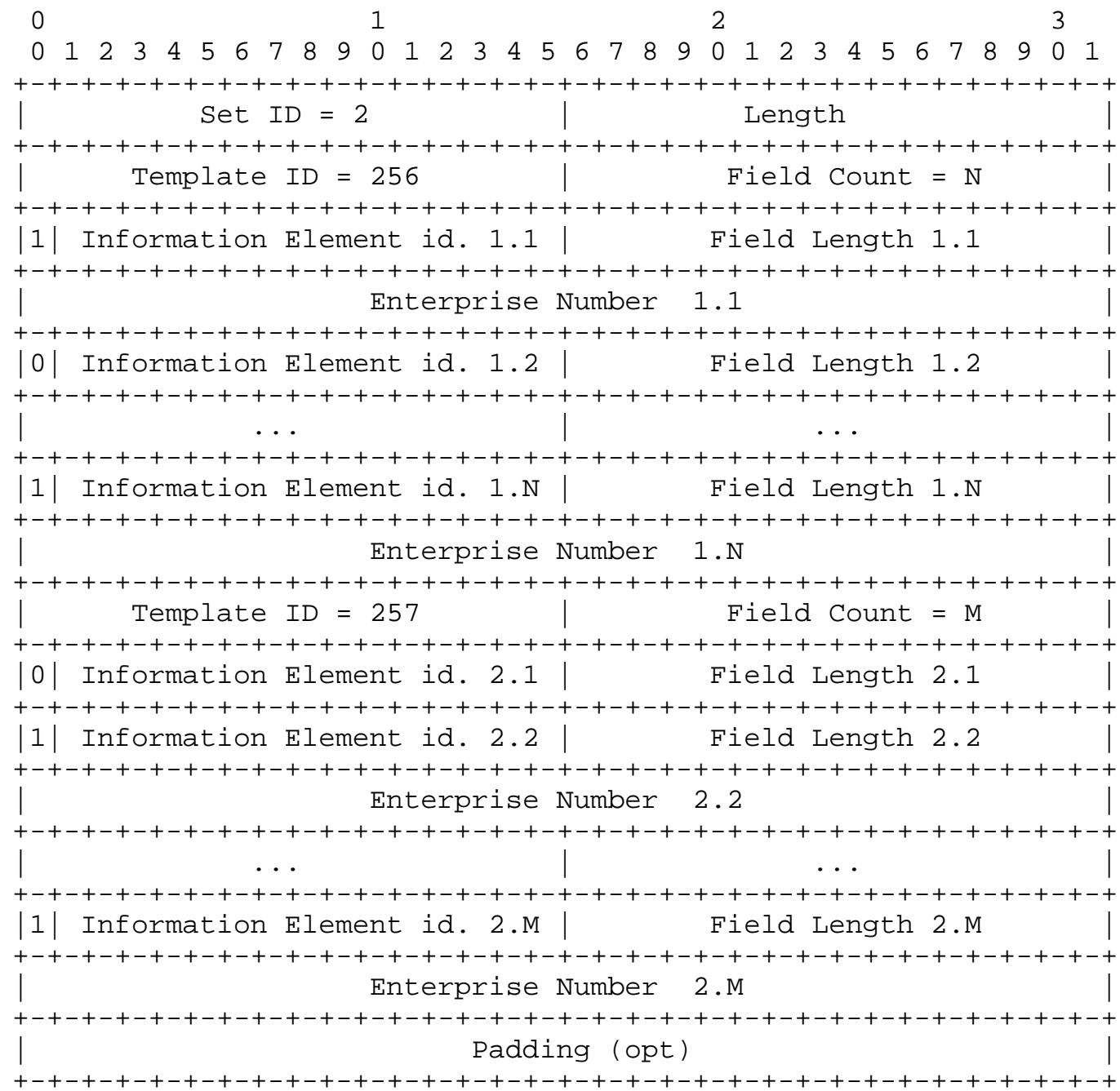

Figure L: Template set Example

Information Element Identifiers 1.2 and 2.1 are defined by the IETF (Enterprise bit $=0$ ) and, therefore, do not need an Enterprise Number to identify them.

\subsubsection{Options Template Record Format}

Thanks to the notion of scope, The Options Template Record gives the Exporter the ability to provide additional information to the collector that would not be possible with Flow Records alone. 
One Options Template Record example is the "Flow Keys", which reports the Flow Keys for a Template, which is defined as the scope. Another example is the "Template configuration", which reports the configuration sampling parameter(s) for the Template, which is defined as the scope.

3.4.2.1. Scope

The scope, which is only available in the Options Template set, gives the context of the reported Information Elements in the Data Records. Note that the IPFIX Message Header already contains the Observation Domain ID (the identifier of the Observation Domain). If not zero, this Observation Domain ID can be considered as an implicit scope for the Data Records in the IPFIX Message. The Observation Domain ID MUST be zero when the IPFIX Message contains Data Records with different Observation Domain ID values defined as scopes.

Multiple Scope Fields MAY be present in the Options Template Record, in which case, the composite scope is the combination of the scopes. For example, if the two scopes are defined as "metering process" and "template", the combined scope is this Template for this Metering Process. The order of the scope Fields, as defined in the Options Template Record, is irrelevant in this case. However, if the order of the Scope Fields in the Options Template Record is relevant, the order of the Scope Fields MUST be used. For example, if the first scope defines the filtering function, while the second scope defines the sampling function, the order of the scope is important. Applying the sampling function first, followed by the filtering function, would lead to potentially different Data Records than applying the filtering function first, followed by the sampling function. In this case, the collector deduces the function order by looking at the order of the scope in the Options Template Record.

The scope is an Information Element specified in the IPFIX Information Model [RFC5102]. An IPFIX-compliant implementation of the Collecting Process SHOULD support this minimum set of Information Elements as scope: LineCardId, TemplateId, exporterIPv4Address, exporterIPv6Address, and ingressInterface. Note that other Information Elements, such as meteringProcessId, exportingProcessId, observationDomainId, etc. are also valid scopes. The IPFIX protocol doesn't prevent the use of any Information Elements for scope. However, some Information Element types don't make sense if specified as scope; for example, the counter Information Elements.

Finally, note that the scope Field Count MUST NOT be zero. 


\subsubsection{Options Template Record Format}

An Options Template Record contains any combination of IANA-assigned and/or enterprise-specific Information Elements identifiers.

The format of the Options Template Record is shown in Figure M. It consists of an Options Template Record Header and one or more Field Specifiers. The definition of the Field specifiers is given in Figure G above.

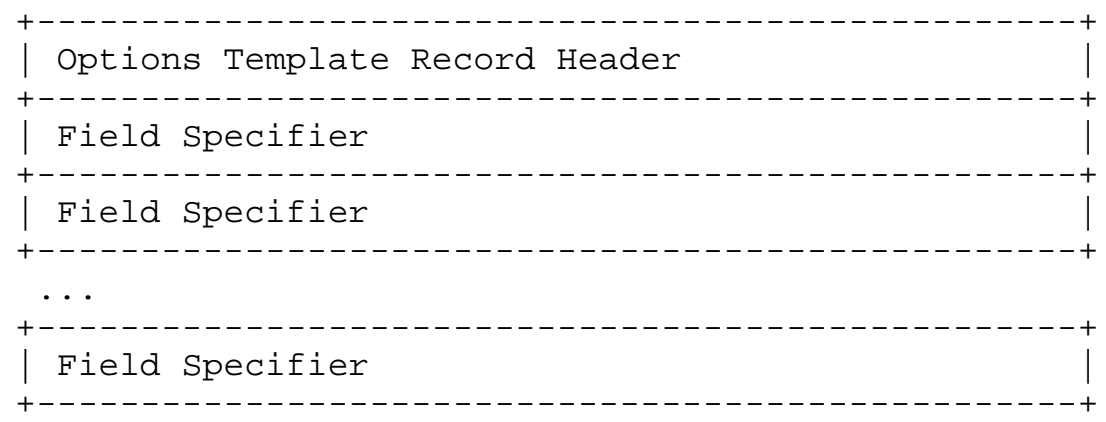

Figure M: Options Template Record Format

The format of the Options Template Record Header is shown in Figure N.

$\begin{array}{lllllll}0 & 1 & 2 & 3\end{array}$

$\begin{array}{llllllllllllllllllllllllllllllll}0 & 1 & 2 & 3 & 4 & 5 & 6 & 7 & 8 & 9 & 0 & 1 & 2 & 3 & 4 & 5 & 6 & 7 & 8 & 9 & 0 & 1 & 2 & 3 & 4 & 5 & 6 & 7 & 8 & 9 & 0 & 1\end{array}$ +-+-+-+-+-+-+-+-+-+-+-+-+-+-+-+-+-+-+-+-+-+-+-+-+-+-+-+-+-+-+-+-+ Template ID $(>255)$
Scope Field Count

Figure N: Options Template Record Header Format

The Options Template Record Header Field Definitions are as follows: Template ID

Template ID of this Options Template Record. This value is greater than 255 . 
Field Count

Number of all fields in this Options Template Record, including the Scope Fields.

Scope Field Count

Number of scope fields in this Options Template Record. The Scope Fields are normal Fields except that they are interpreted as scope at the Collector. The Scope Field Count MUST NOT be zero.

The example in Figure O shows an Option Template set with mixed IETF and enterprise-specific Information Elements. It consists of a set Header, an Option Template Header, and several Field Specifiers.

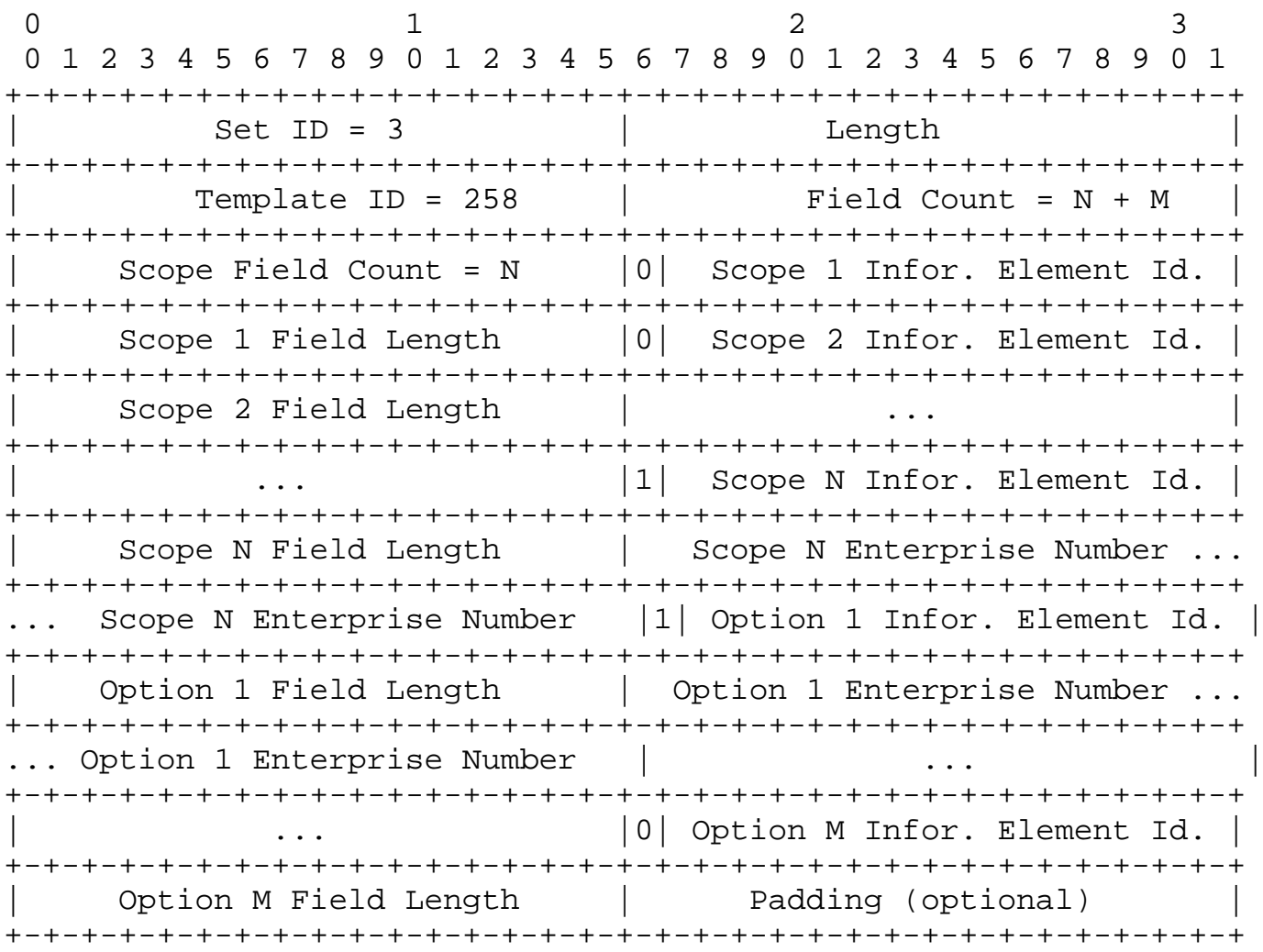

Figure 0: Option Template set Example 


\subsubsection{Data Record Format}

The Data Records are sent in Data Sets. The format of the Data Record is shown in Figure P. It consists only of one or more Field Values. The Template ID to which the Field Values belong is encoded in the set Header field "Set ID", i.e., "Set ID" = "Template ID".

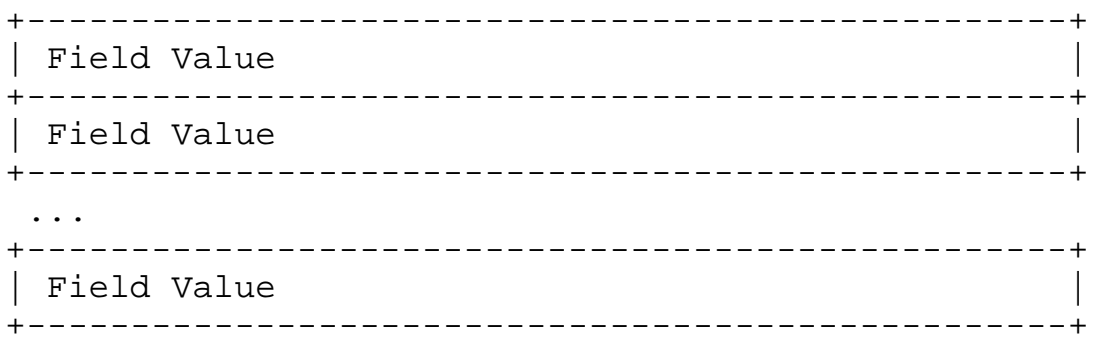

Figure P: Data Record Format

Note that Field Values do not necessarily have a length of 16 bits. Field Values are encoded according to their data type specified in [RFC5102].

Interpretation of the Data Record format can be done only if the Template Record corresponding to the Template ID is available at the Collecting Process.

The example in Figure $Q$ shows a Data Set. It consists of a Set Header and several Field Values. 


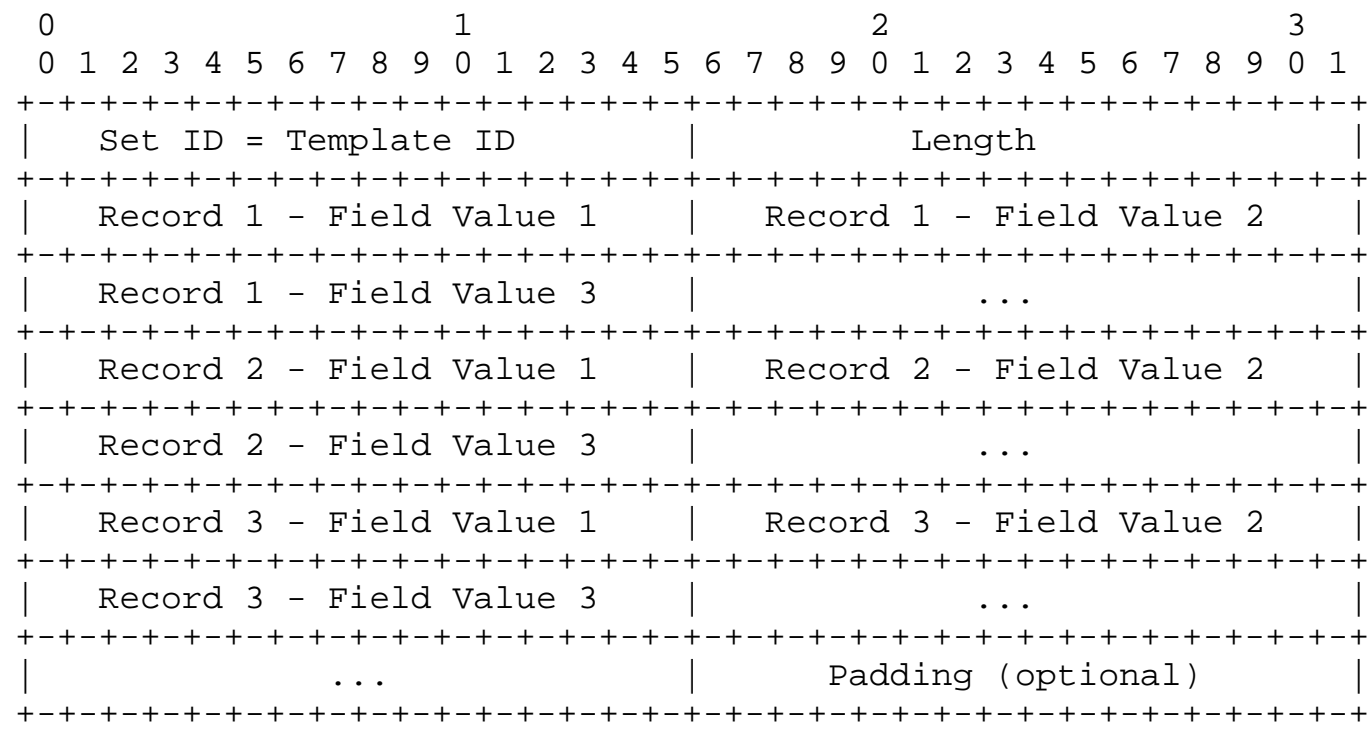

Figure Q: Data Set, Containing Data Records

4. Specific Reporting Requirements

Some specific Options Templates and Options Template Records are necessary to provide extra information about the Flow Records and about the Metering Process.

The Option Template and Options Template Records defined in these subsections, which impose some constraints on the Metering Process and Exporting Process implementations, MAY be implemented. If implemented, the specific Option Templates SHould be implemented as specified in these subsections.

The minimum set of Information Elements is always specified in these Specific IPFIX Options Templates. Nevertheless, extra Information Elements may be used in these specific Options Templates.

\subsection{The Metering Process Statistics Option Template}

The Metering Process Statistics Option Template specifies the structure of a Data Record for reporting Metering Process statistics. It SHOULD contain the following Information Elements that are defined in $[\mathrm{RFC} 5102]$ : 
observationDomainId

An identifier of an Observation Domain that is locally unique to the Exporting Process. This Information Element MUST be defined as a Scope Field.

exportedMessageTotalcount

The total number of IPFIX Messages that the Exporting Process successfully sent to the Collecting Process since the Exporting Process re-initialization.

exportedFlowTotalcount

The total number of Flow Records that the Exporting Process successfully sent to the Collecting Process since the Exporting Process re-initialization.

exportedOctetTotalcount

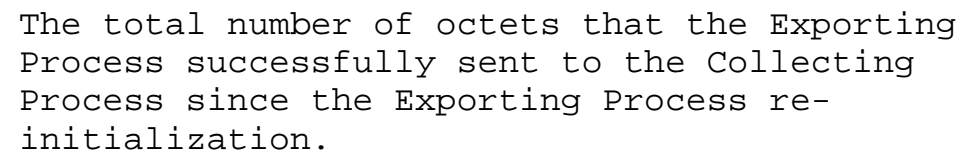

The Exporting Process SHOULD export the Data Record specified by the Metering Process Statistics Option Template on a regular basis or based on some export policy. This periodicity or export policy SHOULD be configurable.

Note that if several Metering Processes are available on the Exporter Observation Domain, the Information Element meteringProcessId MUST be specified as an additional scope Field.

\subsection{The Metering Process Reliability Statistics Option Template}

The Metering Process Reliability option Template specifies the structure of a Data Record for reporting lack of reliability in the Metering Process. It SHOULD contain the following Information Elements that are defined in [RFC5102]:

observationDomainId

An identifier of an Observation Domain that is locally unique to the Exporting Process. This Information Element MUST be defined as a Scope Field. 
ignoredPacketTotalcount

The total number of IP packets that the Metering Process did not process.

ignoredOctet Totalcount

The total number of octets in observed IP packets that the Metering Process did not process.

time first ignored

The timestamp of the first IP packet that was ignored by the Metering Process. For this timestamp, any of the "flowstart" timestamp Information Elements flowStartMilliseconds, flowStartMicroseconds, flowstartNanoseconds, and flowstartDeltaMicroseconds can be used.

time last ignored

The timestamp of the last IP packet that was ignored by the Metering Process. For this timestamp, any of the "flowEnd" timestamp Information Elements flowEndMilliseconds, flowEndMicroseconds, flowEndNanoseconds, and flowEndDeltaMicroseconds can be used.

The Exporting Process SHould export the Data Record specified by the Metering Process Reliability Statistics Option Template on a regular basis or based on some export policy. This periodicity or export policy SHOULD be configurable.

Note that if several Metering Processes are available on the Exporter Observation Domain, the Information Element meteringProcessId MUST be specified as an additional scope Field.

4.3. The Exporting Process Reliability Statistics option Template The Exporting Process Reliability Option Template specifies the structure of a Data Record for reporting lack of reliability in the Exporting process. It SHOULD contain the following Information Elements that are defined in [RFC5102]:

Exporting Process ID

The identifier of the Exporting Process for which lack of reliability is reported. There are three Information Elements specified in [RFC5102] that can be used for this purpose: exporterIPv4Address, exporterIPv6Address, or 
exportingProcessId. This Information Element MUST be defined as a Scope Field.

notsentFlowTotalcount

The total number of Flows that were generated by the Metering Process and dropped by the Metering Process or by the Exporting Process instead of being sent to the collecting Process.

not SentPacketTotalcount

The total number of packets in Flow Records that were generated by the Metering Process and dropped by the Metering Process or by the Exporting Process instead of being sent to the Collecting Process.

notSentoctet Totalcount

The total number of octets in packets in Flow Records that were generated by the Metering Process and dropped by the Metering Process or by the Exporting Process instead of being sent to the Collecting Process.

time first flow dropped

The timestamp of the first Flow was dropped by the Metering Process. For this timestamp, any of the "flowstart" timestamp Information Elements flowStartMilliseconds, flowStartMicroseconds, flowstartNanoseconds, and flowstartDeltaMicroseconds can be used.

time last flow dropped
The timestamp of the last IP packet that was ignored by the Metering Process. For this timestamp, any of the "flowEnd" timestamp Information Elements flowEndMilliseconds, flowEndMicroseconds, flowEndNanoseconds, and flowEndDeltaMicroseconds can be used.

The Exporting Process SHOULD export the Data Record specified by the Exporting Process Reliability Statistics option Template on a regular basis or based on some export policy. This periodicity or export policy SHOULD be configurable.

\subsection{The Flow Keys Option Template}

The Flow Keys Option Template specifies the structure of a Data Record for reporting the Flow Keys of reported Flows. A Flow Keys 
Data Record extends a particular Template Record that is referenced by its templateId identifier. The Template Record is extended by specifying which of the Information Elements contained in the corresponding Data Records describe Flow properties that serve as Flow Keys of the reported Flow.

The Flow Keys Option Template SHould contain the following Information Elements that are defined in [RFC5102]:

templateId An identifier of a Template. This Information Element MUST be defined as a Scope Field.

flowkeyIndicator Bitmap with the positions of the Flow Keys in the Data Records.

5. IPFIX Message Header "Export Time" and Flow Record Time

The IPFIX Message Header "Export Time" field is the time in seconds since 0000 UTC Jan 1, 1970, at which the IPFIX Message Header leaves the Exporter. The time-related Information Elements specified in [RFC5102] MAY use this "Export Time" as base time and specify an offset relative to it, instead of using a common base time, such as 0000 UTC Jan 1, 1970. All Information Elements that do not have their base time defined by their data type MUST have the base time clearly specified in their description.

For example, Data Records requiring a microsecond precision can export the flow start and end times with the flowstartMicroseconds and flowEndMicroseconds Information Elements [RFC5102], containing the time since 0000 UTC Jan 1, 1970. An alternate solution is to export the flowstartDeltaMicroseconds and flowEndDeltaMicroseconds Information Elements [RFC5102] in the Data Record, which respectively report the flow start and end time offsets compared to the IPFIX Message Header "Export Time". The latter solution lowers the export bandwidth requirement while it increases the load on the Exporter, as the Exporting Process must calculate the flowstartDeltaMicroseconds and flowEndDeltaMicroseconds of every single Data Record before exporting the IPFIX Message.

It must be noted that using time-related Information Elements with offset times, compared to the IPFIX Message Header "Export Time", imposes some time constraints on the Data Records contained in the IPFIX Message. In the example of flowstartDeltaMicroseconds and flowEndDeltaMicroseconds Information Elements [RFC5102], the Data Record must be exported within a maximum of 71 minutes after its creation. Otherwise, the 32-bit counter would not be sufficient to contain the flow start time offset. 
6. Linkage with the Information Model

The Information Elements [RFC5102] MUST be sent in canonical format in network-byte order (also known as the big-endian byte ordering).

\subsection{Encoding of IPFIX Data Types}

The following sections will define the encoding of the data types specified in [RFC5102].

\subsubsection{Integral Data Types}

Integral data types -- octet, signed8, unsigned16, signed16, unsigned32, signed32, signed64, and unsigned64-- MUST be encoded using the default canonical format in network-byte order. Signed Integral data types are represented in two's complement notation.

\subsubsection{Address Types}

Address types -- macAddress, ipv4Address, and ipv6Address -- MUST be encoded the same way as the integral data types. The macAddress is treated as a 6-octet integer, the ipv4Address as a 4-octet integer, and the ipv6Address as a 16-octet integer.

6.1.3. float 32

The float32 data type MUST be encoded as an IEEE single-precision 32-bit floating point-type, as specified in [IEEE.754.1985].

\subsection{4. float 64}

The float 64 data type MUST be encoded as an IEEE double-precision 64-bit floating point-type, as specified in [IEEE.754.1985].

\subsection{5. boolean}

The boolean data type is specified according to the Truthvalue in [RFC2579]: it is an integer with the value 1 for true and a value 2 for false. Every other value is undefined. The boolean data type MUST be encoded in a single octet.

6.1.6. string and octetarray

The data type string represents a finite length string of valid characters of the Unicode character encoding set. The string data type MUST be encoded in UTF-8 format. The string is sent as an array of octets using an Information Element of fixed or variable length. 
The length of the Information Element specifies the length of the octetarray.

\subsection{7. dateTimeSeconds}

The data type dateTimeseconds represents a time value in units of seconds normalized to the GMT timezone. It MUST be encoded in a 32-bit integer containing the number of seconds since 0000 UTC Jan 1, 1970. The 32-bit integer allows the time encoding up to 136 years.

\subsection{8. dateTimeMilliseconds}

The data type dateTimeMilliseconds represents a time value in units of milliseconds normalized to the GMT timezone. It MUST be encoded in a 64-bit integer containing the number of milliseconds since 0000 UTC Jan 1, 1970 .

\subsection{9. dateTimeMicroseconds}

The data type dateTimeMicroseconds represents a time value in units of microseconds normalized to the GMT timezone. It MUST be encoded in a 64-bit integer, according to the NTP format given in [RFC1305].

\subsubsection{0. dateTimeNanoseconds}

The data type of dateTimeNanoseconds represents a time value in units of nanoseconds normalized to the GMT time zone. It MUST be encoded in a 64-bit integer, according to the NTP format given in [RFC1305].

\subsection{Reduced Size Encoding of Integer and Float Types}

Information Elements containing integer, string, float, and octetarray types in the information model MAY be encoded using fewer octets than those implied by their type in the information model definition [RFC5102], based on the assumption that the smaller size is sufficient to carry any value the Exporter may need to deliver. This reduces the network bandwidth requirement between the Exporter and the collector. Note that the Information Element definitions [RFC5102] will always define the maximum encoding size.

For instance, the information model [RFC5102] defines bytecount as an unsigned64 type, which would require 64 bits. However, if the Exporter will never locally encounter the need to send a value larger than 4294967295, it may chose to send the value instead as an unsigned32. For example, a core router would require an unsigned64 bytecount, while an unsigned32 might be sufficient for an access router. 
This behavior is indicated by the Exporter by specifying a type size with a smaller length than that associated with the assigned type of the Information Element. In the example above, the Exporter would place a length of 4 versus 8 in the Template.

If reduced sizing is used, it MUST only be applied to the following integer types: unsigned64, signed64, unsigned32, signed32, unsigned16, and signed16. The signed versus unsigned property of the reported value MUST be preserved. The reduction in size can be to any number of octets smaller than the original type if the data value still fits, i.e., so that only leading zeroes are dropped. For example, an unsigned64 can be reduced in size to $7,6,5,4,3,2$, or 1 octet $(\mathrm{s})$.

Reduced sizing can also be used to reduce float64 to float 32 . The float32 not only has a reduced number range, but due to the smaller mantissa, is also less precise.

The reduced size encoding MUST NOT be applied to dateTimeMicroseconds or to dateTimeNanoseconds because these represent an inherent structure that would be destroyed by using less than the original number of bytes.

7. Variable-Length Information Element

The IPFIX Template mechanism is optimized for fixed-length Information Elements [RFC5102]. Where an Information Element has a variable length, the following mechanism MUST be used to carry the length information for both the IETF and proprietary Information Elements.

In the Template Set, the Information Element Field Length is recorded as 65535. This reserved length value notifies the Collecting Process that length of the Information Element will be carried in the Information Element content itself.

In most cases, the length of the Information Element will be less than 255 octets. The following length-encoding mechanism optimizes the overhead of carrying the Information Element length in this majority case. The length is carried in the octet before the Information Element, as shown in Figure $\mathrm{R}$. 


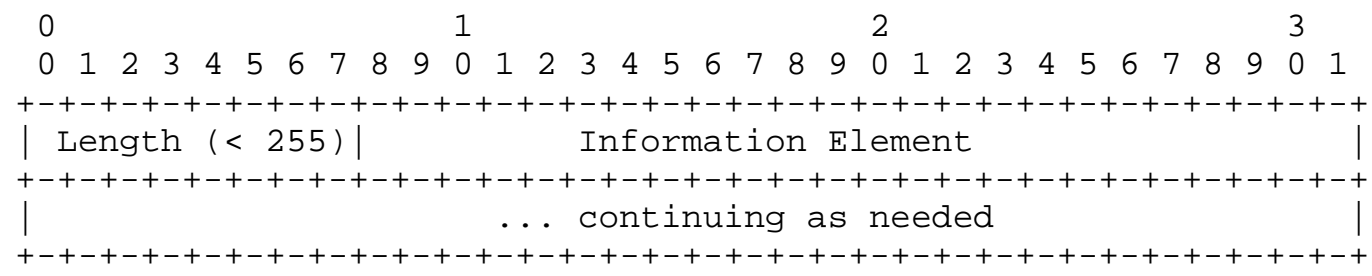

Figure R: Variable-Length Information Element (length < 255 octets)

If the length of the Information Element is greater than or equal to 255 octets, the length is encoded into 3 octets before the Information Element. The first octet is 255, and the length is carried in the second and third octets, as shown in Figure $S$.

$\begin{array}{lllllllllllll}0 & 1 & 2 & & & \end{array}$

$\begin{array}{llllllllllllllllllllllllllllllll}0 & 1 & 2 & 3 & 4 & 5 & 6 & 7 & 8 & 9 & 0 & 1 & 2 & 3 & 4 & 5 & 6 & 7 & 8 & 9 & 0 & 1 & 2 & 3 & 4 & 5 & 6 & 7 & 8 & 9 & 0 & 1\end{array}$

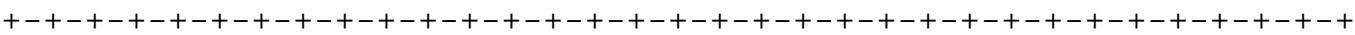
255
$\mid$

Figure S: Variable-Length Information Element (length 0 to 65535 octets)

The octets carrying the length (either the first or the first three octets) MUST NOT be included in the length of the Information Element.

8. Template Management

This section describes Template Management when using SCTP and PR-SCTP as the transport protocol. Any necessary changes to Template Management specifically related to TCP or UDP transport protocols are specified in Section 10.

The Exporting Process assigns and maintains the Template IDs per SCTP association for the Exporter's Observation Domains. A newly created Template Record is assigned an unused Template ID by the Exporting Process.

If a specific Information Element is required by a Template, but is not available in observed packets, the Exporting Process MAY choose to export Flow Records without this Information Element in a Data Record defined by a new Template. 
If an Information Element is required more than once in a Template, the different occurrences of this Information Element SHoULD follow the logical order of their treatments by the Metering Process. For example, if a selected packet goes through two hash functions, and if the two hash values are sent within a single Template, the first occurrence of the hash value should belong to the first hash function in the Metering Process. For example, when exporting the two source IP addresses of an IPv4 in IPv4 packets, the first sourceIPv4Address Information Element occurrence should be the IPv4 address of the outer header, while the second occurrence should be the inner header one.

Template Sets and Options Template Sets may be sent on any SCTP stream. Template sets and Options Template sets MUST be sent reliably, using SCTP-ordered delivery. As such, the collecting Process MUST store the Template Record information for the duration of the SCTP association so that it can interpret the corresponding Data Records that are received in subsequent Data Sets.

The Exporting Process SHOULD transmit the Template set and Options Template set in advance of any Data sets that use that (Options) Template ID, to help ensure that the Collector has the Template Record before receiving the first Data Record. Data Records that correspond to a Template Record MAY appear in the same and/or subsequent IPFIX Message $(s)$.

Different Observation Domains from the same SCTP association may use the same Template ID value to refer to different Templates.

The Templates that are not used anymore SHOULD be deleted. Before reusing a Template ID, the Template MUST be deleted. In order to delete an allocated Template, the Template is withdrawn through the use of a Template Withdrawal Message.

The Template Withdrawal Message MUST NOT be sent until sufficient time has elapsed to allow the collecting Process to receive and process the last Data Record using this Template information. This time MUST be configurable. A suitable default value is 5 seconds after the last Data Record has been sent.

The Template ID from a withdrawn Template MUST NOT be reused until sufficient time has elapsed to allow for the Collecting Process to receive and process the Template Withdrawal Message.

A Template Withdrawal Message is a Template Record for that Template ID with a Field Count of 0 . The format of the Template Withdrawal Message is shown in Figure $\mathrm{T}$. 


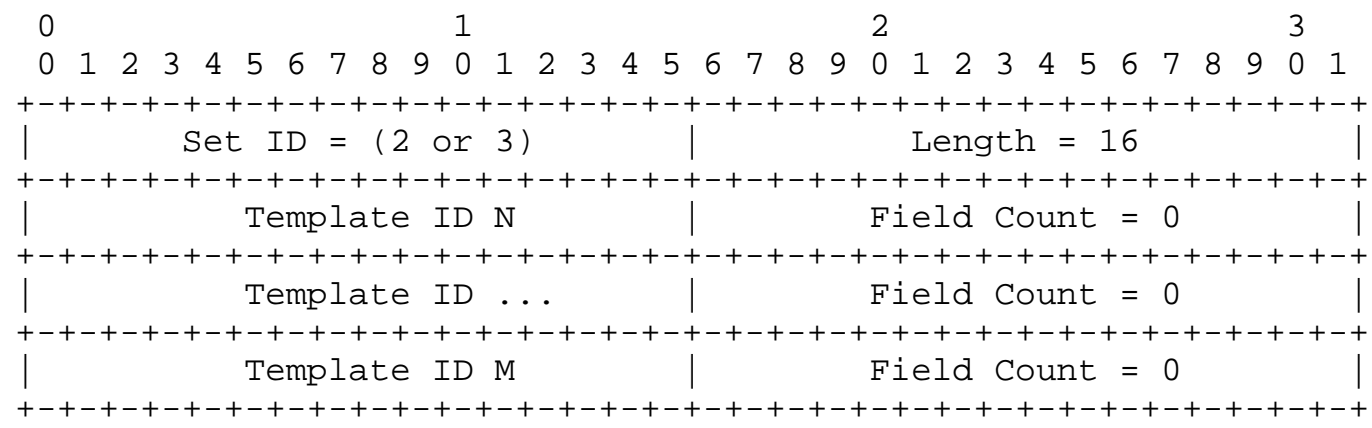

Figure T: Template Withdrawal Message Format

The Set ID field MUST contain the value 2 for Template Set Withdrawal and the value 3 for Options Template set Withdrawal. Multiple Template IDs MAY be withdrawn with a single Template Withdrawal Message, in that case, padding MAY be used.

The Template Withdrawal Message withdraws the Template IDs for the Observation Domain ID specified in the IPFIX Message Header.

The Template Withdrawal Message may be sent on any SCTP stream. The Template Withdrawal Message MUST be sent reliably, using SCTP-ordered delivery .

The Template Withdrawal Message MUST NOT contain new Template or Options Template Records.

If the measurement parameters change such that a new Template is required, the Template MUST be withdrawn (using a Template Withdraw Message and a new Template definition) or an unused Template ID MUST be used. Examples of the measurement changes are: a new sampling rate, a new Flow expiration process, a new filtering definition, etc.

When the SCTP association shuts down or the Exporting Process restarts, all Template assignments are lost and Template IDs MUST be reassigned.

If the Metering Process restarts, the Exporting Process MUST either reuse the previously assigned Template ID for each Template, or it MUST withdraw the previously issued Template IDs by sending Template Withdraw Message(s) before reusing them.

A Template Withdrawal Message to withdraw all Templates for the Observation Domain ID specified in the IPFIX Message Header MAY be used. Its format is shown in Figure $U$. 


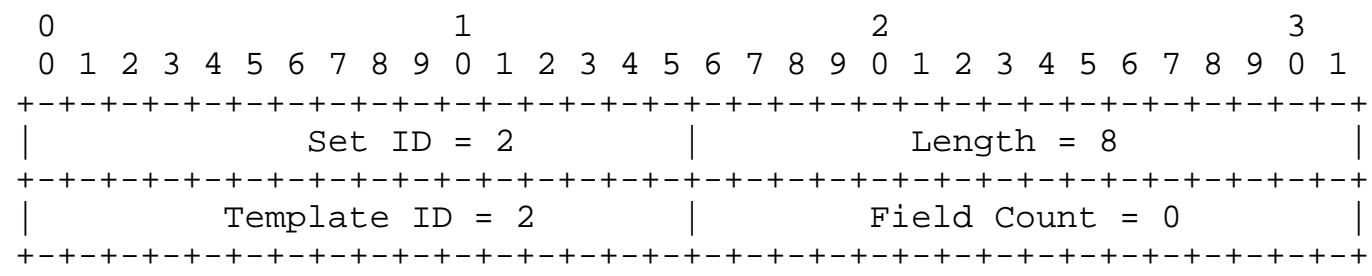

Figure U: All Data Templates Withdrawal Message Format

A Template Withdrawal Message to withdraw all Options Templates for the Observation Domain ID specified in the IPFIX Message Header MAY be used. Its format is shown in Figure V.

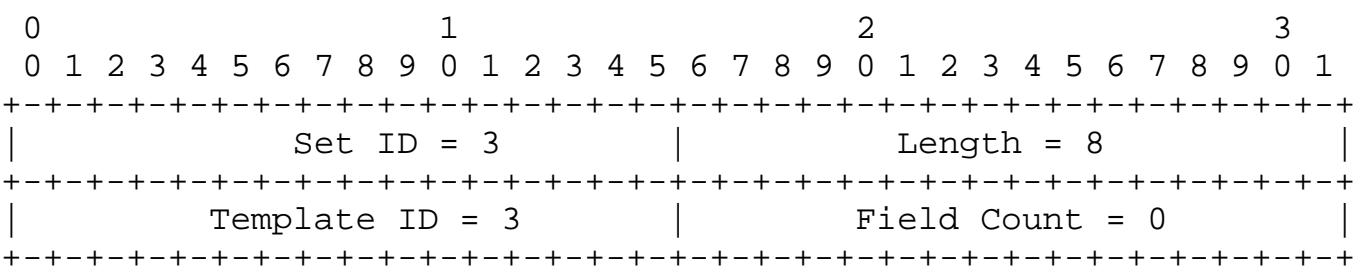

Figure V: All Options Templates Withdrawal Message Format

When the SCTP association restarts, the Exporting Process MUST resend all the Template Records.

9. The Collecting Process's Side

This section describes the Collecting Process when using SCTP and PR-SCTP as the transport protocol. Any necessary changes to the Collecting Process specifically related to TCP or UDP transport protocols are specified in section 10 .

The Collecting Process SHOULD listen for a new association request from the Exporting Process. The Exporting Process will request a number of streams to use for export. An Exporting Process MAY request and support more than one stream per SCTP association.

If the Collecting Process receives a malformed IPFIX Message, it MUST reset the SCTP association, discard the IPFIX Message, and SHOULD log the error. Note that non-zero set padding does not constitute a malformed IPFIX Message.

Template Sets and Option Template Sets are only sent once. The Collecting Process MUST store the Template Record information for the duration of the association so that it can interpret the corresponding Data Records that are received in subsequent Data sets. 
Template IDs are unique per SCTP association and per Observation Domain. If the Collecting Process receives a Template that has already been received but that has not previously been withdrawn (i.e., a Template Record from the same Exporter Observation Domain with the same Template ID received on the SCTP association), then the Collecting Process MUST shut down the association.

When an SCTP association is closed, the collecting Process MUST discard all Templates received over that association and stop decoding IPFIX Messages that use those Templates.

The Collecting Process normally receives Template Records from the Exporting Process before receiving Data Records. The Data Records are then decoded and stored by the collector. If the Template Records have not been received at the time Data Records are received, the Collecting Process MAY store the Data Records for a short period of time and decode them after the Template Records are received. A Collecting Process MUST NOT assume that the Data set and the associated Template Set (or Options Template Set) are exported in the same IPFIX Message.

The Collecting Process MUST note the Information Element identifier of any Information Element that it does not understand and MAY discard that Information Element from the Flow Record.

The Collector MUST accept padding in Data Records and Template Records. The padding size is the set Length minus the size of the Set Header (4 octets for the set ID and the set Length), modulo the Record size deduced from the Template Record.

The IPFIX protocol has a Sequence Number field in the Export header that increases with the number of IPFIX Data Records in the IPFIX Message. A Collector may detect out-of-sequence, dropped, or duplicate IPFIX Messages by tracking the Sequence Number. A Collector SHOULD provide a logging mechanism for tracking out-of-sequence IPFIX Messages. Such out-of-sequence IPFIX Messages may be due to Exporter resource exhaustion where it cannot transmit messages at their creation rate, an Exporting Process reset, congestion on the network link between the Exporter and Collector, Collector resource exhaustion where it cannot process the IPFIX Messages at their arrival rate, out-of-order packet reception, duplicate packet reception, or an attacker injecting false messages.

If a Collecting Process receives a Template Withdrawal Message, the Collecting Process MUST delete the corresponding Template Records associated with the specific SCTP association and specific Observation Domain, and stop decoding IPFIX Messages that use the withdrawn Templates. 
If the Collecting Process receives a Template Withdraw message for a Template Record it has not received before on this SCTP association, it MUST reset the SCTP association, discard the IPFIX Message, and SHOULD log the error as it does for malformed IPFIX Messages.

A Collecting Process that receives IPFIX Messages from several Observation Domains on the same Transport Session MUST be aware that the uniqueness of the Template ID is not guaranteed across observation Domains.

The Collector MUST support the use of Templates containing multiple occurrences of the similar Information Elements.

10. Transport Protocol

The IPFIX Protocol Specification has been designed to be transport protocol independent. Note that the Exporter can export to multiple Collecting Processes using independent transport protocols.

The IPFIX Message Header 16-bit Length field limits the length of an IPFIX Message to 65535 octets, including the header. A Collecting Process MUST be able to handle IPFIX Message lengths of up to 65535 octets.

10.1. Transport Compliance and Transport Usage

We need to differentiate between what must be implemented (so that operators can interoperably deploy compliant implementations from different vendors) and what should or could be used in various operational environments. We must also make sure that ALL implementations can operate in a congestion-aware and congestion-avoidance mode.

SCTP [RFC4960] using the PR-SCTP extension specified in [RFC3758] MUST be implemented by all compliant implementations. UDP [UDP] MAY also be implemented by compliant implementations. TCP [TCP] MAY also be implemented by compliant implementations.

PR-SCTP SHOULD be used in deployments where Exporters and Collectors are communicating over links that are susceptible to congestion. PR-SCTP is capable of providing any required degree of reliability.

TCP MAY be used in deployments where Exporters and Collectors communicate over links that are susceptible to congestion, but PR-SCTP is preferred due to its ability to limit back pressure on Exporters and its message versus stream orientation. 
UDP MAY be used, although it is not a congestion-aware protocol. However, the IPFIX traffic between Exporter and Collector MUST run in an environment where IPFIX traffic has been provisioned for, or is contained through some other means.

10.2. $\operatorname{SCTP}$

This section describes how IPFIX can be transported over SCTP [RFC4960] using the PR-SCTP [RFC3758] extension.

\subsubsection{Congestion Avoidance}

The SCTP transport protocol provides the required level of congestion avoidance by design.

SCTP will detect congestion in the end-to-end path between the IPFIX Exporting Process and the IPFIX Collecting Process, and limit the transfer rate accordingly. When an IPFIX Exporting Process has records to export, but detects that transmission by SCTP is temporarily impossible, it can either wait until sending is possible again, or it can decide to drop the record. In the latter case, the dropped export data MUST be accounted for, so that the amount of dropped export data can be reported.

10.2.2. Reliability

The SCTP transport protocol is by default reliable, but has the capability to deliver messages with partial reliability [RFC3758].

Using reliable SCTP messages for the IPFIX export is not in itself a guarantee that all Data Records will be delivered. If there is congestion on the link from the Exporting Process to the Collecting Process, or if a significant number of retransmissions are required, the send queues on the Exporting Process may fill up; the Exporting Process MAY either suspend, export, or discard the IPFIX Messages. If Data Records are discarded the IPFIX Sequence Numbers used for export MUST reflect the loss of data.

10.2.3. MTU

SCTP provides the required IPFIX Message fragmentation service based on path MTU discovery. 


\subsubsection{Exporting Process}

\subsubsection{Association Establishment}

The IPFIX Exporting Process SHOULD initiate an SCTP association with the IPFIX Collecting Process. By default, the Collecting Process listens for connections on SCTP port 4739. By default, the Collecting Process listens for secure connections on SCTP port 4740 (refer to the Security Considerations section). By default, the Exporting Process tries to connect to one of these ports. It MUST be possible to configure both the Exporting and Collecting Processes to use a different SCTP port.

The Exporting Process MAY establish more than one association (connection "bundle" in SCTP terminology) to the Collecting Process.

An Exporting Process MAY support more than one active association to different Collecting Processes (including the case of different Collecting Processes on the same host).

\subsubsection{Association Shutdown}

When an Exporting Process is shut down, it SHOULD shut down the SCTP association.

When a Collecting Process no longer wants to receive IPFIX Messages, it SHOULD shut down its end of the association. The collecting Process SHOULD continue to receive and process IPFIX Messages until the Exporting Process has closed its end of the association.

When a collecting Process detects that the SCTP association has been abnormally terminated, it MUST continue to listen for a new association establishment.

When an Exporting Process detects that the SCTP association to the Collecting Process is abnormally terminated, it SHould try to re-establish the association.

Association timeouts SHOULD be configurable.

10.2.4.3. Stream

An Exporting Process MAY request more than one SCTP stream per association. Each of these streams may be used for the transmission of IPFIX Messages containing Data Sets, Template Sets, and/or Options Template Sets. 
Depending on the requirements of the application, the Exporting Process may send Data sets with full or partial reliability, using ordered or out-of-order delivery, over any SCTP stream established during SCTP Association setup.

An IPFIX Exporting Process MAY use any PR-SCTP Service Definition as per Section 4 of the PR-SCTP [RFC3758] specification when using partial reliability to transmit IPFIX Messages containing only Data Sets.

However, Exporting Processes SHOULD mark such IPFIX Messages for retransmission for as long as resource or other constraints allow.

10.2.4.4. Template Management

When the transport protocol is SCTP, the default Template Management described in section 8 is used.

10.2.5. Collecting Process

When the transport protocol is SCTP, the default Collector processing described in section 9 is used.

10.2.6. Failover

If the Collecting Process does not acknowledge the attempt by the Exporting Process to establish an association, the Exporting Process should retry using the SCTP exponential backoff feature. The Exporter MAY log an alarm if the time to establish the association exceeds a specified threshold, configurable on the Exporter.

If Collecting Process failover is supported by the Exporting Process, a second SCTP association MAY be opened in advance.

10.3. UDP

This section describes how IPFIX can be transported over UDP [UDP] .

10.3.1. Congestion Avoidance

UDP has no integral congestion-avoidance mechanism. Its use over congestion-sensitive network paths is therefore not recommended. UDP MAY be used in deployments where Exporters and Collectors always communicate over dedicated links that are not susceptible to congestion, i.e., over provisioned links compared to the maximum export rate from the Exporters. 


\subsubsection{Reliability}

UDP is not a reliable transport protocol, and cannot guarantee delivery of messages. IPFIX Messages sent from the Exporting Process to the Collecting Process using UDP may therefore be lost. UDP MUST NOT be used unless the application can tolerate some loss of IPFIX Messages.

The Collecting Process SHOULD deduce the loss and reordering of IPFIX Data Records by looking at the discontinuities in the IPFIX Sequence Number. In the case of UDP, the IPFIX Sequence Number contains the total number of IPFIX Data Records sent for the UDP Transport Session prior to the receipt of this IPFIX Message, modulo 2^32. A Collector SHOULD detect out-of-sequence, dropped, or duplicate IPFIX Messages by tracking the Sequence Number. Templates sent from the Exporting Process to the Collecting Process using UDP as a transport MUST be re-sent at regular intervals, in case previous copies were lost.

\subsubsection{MTU}

The maximum size of exported messages MUST be configured such that the total packet size does not exceed the path MTU. If the path MTU is unknown, a maximum packet size of 512 octets SHOULD be used.

10.3.4. Port Numbers

By default, the Collecting Process listens on the UDP port 4739. By default, the collecting Process listens for secure connections on UDP port 4740 (refer to the "Security Considerations" section). By default, the Exporting Process tries to connect to one of these ports. It MUST be possible to configure both the Exporting and Collecting Processes to use a different UDP port.

\subsubsection{Exporting Process}

The Exporting Process MAY duplicate the IPFIX Message to the several Collecting Processes.

10.3.6. Template Management

When IPFIX uses UDP as the transport protocol, Template sets and Option Template sets MUST be re-sent at regular intervals. The frequency of the (Options) Template transmission MUST be configurable. The default value for the frequency of the (Options) Template transmission is 10 minutes. The Exporting Process SHOULD transmit the Template set and Options Template set in advance of any Data sets that use that (Options) Template ID to help ensure that the 
Collector has the Template Record before receiving the first Data Record.

In the event of configuration changes, the Exporting Process SHOULD send multiple copies of the new Template definitions, in different IPFIX Messages, at an accelerated rate. In such a case, it SHOULD transmit the changed Template Record(s) and Options Template Record(s), without any data, in advance to help ensure that the collector will have the correct Template information before receiving the first data.

If the Option Template scope is defined in another Template, then both Templates SHOULD be sent in the same IPFIX Message. For example, if a Flow Key Option Template (see Section 4.4) is sent in an option Template, then the associated Template SHould be sent in the same IPFIX Message.

Following a configuration change that can modify the interpretation of the Data Records (for example, a sampling rate change) a new Template ID MUST be used, and the old Template ID MUST NOT be reused until its lifetime (see Section 10.3.7) has expired.

If UDP is selected as the transport protocol, the Template Withdraw Messages MUST NOT be used, as this method is inefficient due to the unreliable nature of UDP.

\subsubsection{Collecting Process}

The Collecting Process MUST associate a lifetime with each Template (or another definition of an identifier considered unique within the Transport Session) received via UDP. Templates (and similar definitions) not refreshed by the Exporting Process within the lifetime are expired at the collecting Process. If the Template (or other definition) is not refreshed before that lifetime has expired, the Collecting Process MUST discard that definition and any current and future associated Data Records. In which case, an alarm MUST be logged. The Collecting Process MUST NOT decode any further Data Records that are associated with the expired Template. If a Template is refreshed with a Template Record that differs from the previously received Template Record, the Collecting Process SHould log a warning and replace the previously received Template Record with the new one. The Template lifetime at the collecting Process MUST be at least 3 times higher than the Template refresh timeout configured on the Exporting Process.

Template IDs are unique per UDP session and per Observation Domain. At any given time, the collecting Process SHOULD maintain the following for all the current Template Records and Options Template 
Records: <IPFIX Device, Exporter source UDP port, Observation Domain ID, Template ID, Template Definition, Last Received>.

The Collecting Process SHOULD accept Data Records without the associated Template Record (or other definitions) required to decode the Data Record. If the Template Records (or other definitions such as Common Properties) have not been received at the time Data Records are received, the Collecting Process SHOULD store the Data Records for a short period of time and decode them after the Template Records (or other definitions) are received. The short period of time MUST be lower than the lifetime of definitions associated with identifiers considered unique within the UDP session.

If the Collecting Process receives a malformed IPFIX Message, it MUST discard the IPFIX Message and SHOULD log the error.

10.3.8. Failover

Because UDP is not a connection-oriented protocol, the Exporting Process is unable to determine from the transport protocol that the Collecting Process is no longer able to receive the IPFIX Messages. Therefore, it cannot invoke a failover mechanism. However, the Exporting Process MAY duplicate the IPFIX Message to several Collecting Processes.

10.4. TCP

This section describes how IPFIX can be transported over TCP [TCP].

10.4.1. Connection Management

10.4.1.1. Connection Establishment

The IPFIX Exporting Process initiates a TCP connection to the Collecting Process. By default, the Collecting Process listens for connections on TCP port 4739. By default, the Collecting Process listens for secure connections on TCP port 4740 (refer to the Security Considerations section). By default, the Exporting Process tries to connect to one of these ports. It MUST be possible to configure both the Exporting Process and the Collecting Process to use a different TCP port.

An Exporting Process MAY support more than one active connection to different Collecting Processes (including the case of different Collecting Processes on the same host).

The Exporter MAY log an alarm if the time to establish the connection exceeds a specified threshold, configurable on the Exporter. 


\subsubsection{Graceful Connection Release}

When an Exporting Process is shut down, it SHOULD shut down the TCP connection.

When a collecting Process no longer wants to receive IPFIX Messages, it SHOULD close its end of the connection. The collecting Process SHOULD continue to read IPFIX Messages until the Exporting Process has closed its end.

\subsubsection{Restarting Interrupted Connections}

When a Collecting Process detects that the TCP connection to the Exporting Process has terminated abnormally, it MUST continue to listen for a new connection.

When an Exporting Process detects that the TCP connection to the Collecting Process has terminated abnormally, it sHould try to re-establish the connection. Connection timeouts and retry schedules SHOULD be configurable. In the default configuration, an Exporting Process MUST NOT attempt to establish a connection more frequently than once per minute.

\subsubsection{Failover}

If the Collecting Process does not acknowledge the attempt by the Exporting Process to establish a connection, it will retry using the TCP exponential backoff feature.

If Collecting Process failover is supported by the Exporting Process, a second TCP connection MAY be opened in advance.

10.4.2. Data Transmission

Once a TCP connection is established, the Exporting Process starts sending IPFIX Messages to the Collecting Process.

\subsubsection{IPFIX Message Encoding}

IPFIX Messages are sent over the TCP connection without any special encoding. The Length field in the IPFIX Message Header defines the end of each IPFIX Message and thus the start of the next IPFIX Message. This means that IPFIX Messages cannot be interleaved.

In the case of TCP, the IPFIX Sequence Number contains the total number of IPFIX Data Records sent from this TCP connection, from the current Observation Domain by the Exporting Process, prior to the receipt of this IPFIX Message, modulo $2^{\wedge} 32$. 
If an Exporting Process exports data from multiple observation Domains, it should be careful to choose IPFIX Message lengths appropriately to minimize head-of-line blocking between different Observation Domains. Multiple TCP connections MAY be used to avoid head-of-line between different Observation Domains.

10.4.2.2. Template Management

For each Template, the Exporting Process MUST send the Template Record before exporting Data Records that refer to that Template.

Template IDs are unique per TCP connection and per observation Domain. A Collecting Process MUST record all Template and Options Template Records for the duration of the connection, as an Exporting Process is not required to re-export Template Records.

When the TCP connection restarts, the Exporting Process MUST resend all the Template Records.

When a TCP connection is closed, the Collecting Process MUST discard all Templates received over that connection and stop decoding IPFIX Messages that use those Templates.

The Templates that are not used anymore SHOULD be deleted. Before reusing a Template ID, the Template MUST be deleted. In order to delete an allocated Template, the Template is withdrawn through the use of a Template Withdrawal Message over the TCP connection.

If the Collecting Process receives a malformed IPFIX Message, it MUST reset the TCP connection, discard the IPFIX Message, and SHOULD log the error.

10.4.2.3. Congestion Handling and Reliability

TCP ensures reliable delivery of data from the Exporting Process to the Collecting Process. TCP also controls the rate at which data can be sent from the Exporting Process to the collecting Process, using a mechanism that takes into account both congestion in the network and the capabilities of the receiver.

Therefore, an IPFIX Exporting Process may not be able to send IPFIX Messages at the rate that the Metering Process generates it, either because of congestion in the network or because the Collecting Process cannot handle IPFIX Messages fast enough. As long as congestion is transient, the Exporting Process can buffer IPFIX Messages for transmission. But such buffering is necessarily limited, both because of resource limitations and because of 
timeliness requirements, so ongoing and/or severe congestion may lead to a situation where the Exporting Process is blocked.

When an Exporting Process has Data Records to export but the transmission buffer is full, and it wants to avoid blocking, it can decide to drop some Data Records. The dropped Data Records MUST be accounted for, so that the amount can later be exported.

When an Exporting Process finds that the rate at which records should be exported is consistently higher than the rate at which TCP sending permits, it should provide back pressure to the Metering Processes. The Metering Process could then adapt by temporarily reducing the amount of data it generates, for example, using sampling or aggregation.

\subsubsection{Collecting Process}

The Collecting Process SHOULD listen for a new TCP connection from the Exporting Process.

If the Collecting Process receives a malformed IPFIX Message, it MUST reset the TCP connection, discard the IPFIX Message, and SHOULD log the error. Note that non-zero set padding does not constitute a malformed IPFIX Message.

Template Sets and Option Template sets are only sent once. The Collecting Process MUST store the Template Record information for the duration of the connection so that it can interpret the corresponding Data Records that are received in subsequent Data sets.

Template IDs are unique per TCP connection and per Observation Domain. If the Collecting Process receives a Template that has already been received but that has not previously been withdrawn (i.e., a Template Record from the same Exporter Observation Domain with the same Template ID received on the TCP connection), then the Collecting Process MUST shut down the connection.

When a TCP connection is closed, the collecting Process MUST discard all Templates received over that connection and stop decoding IPFIX Messages that use those Templates.

If a Collecting Process receives a Template Withdrawal Message, the Collecting Process MUST delete the corresponding Template Records associated with the specific TCP connection and specific Observation Domain, and stop decoding IPFIX Messages that use the withdrawn Templates. 
If the Collecting Process receives a Template Withdrawal Message for a Template Record it has not received before on this TCP connection, it MUST reset the TCP association, discard the IPFIX Message, and SHOULD log the error as it does for malformed IPFIX Messages.

\section{Security Considerations}

The security considerations for the IPFIX protocol have been derived from an analysis of potential security threats, as discussed in the "Security Considerations" section of IPFIX requirements [RFC3917]. The requirements for IPFIX security are as follows:

1. IPFIX must provide a mechanism to ensure the confidentiality of IPFIX data transferred from an Exporting Process to a collecting Process, in order to prevent disclosure of Flow Records transported via IPFIX.

2. IPFIX must provide a mechanism to ensure the integrity of IPFIX data transferred from an Exporting Process to a Collecting Process, in order to prevent the injection of incorrect data or control information (e.g., Templates) into an IPFIX Message stream.

3. IPFIX must provide a mechanism to authenticate IPFIX Collecting and Exporting Processes, to prevent the collection of data from an unauthorized Exporting Process or the export of data to an unauthorized Collecting Process.

Because IPFIX can be used to collect information for network forensics and billing purposes, attacks designed to confuse, disable, or take information from an IPFIX collection system may be seen as a prime objective during a sophisticated network attack.

An attacker in a position to inject false messages into an IPFIX Message stream can either affect the application using IPFIX (by falsifying data), or the IPFIX Collecting Process itself (by modifying or revoking Templates, or changing options); for this reason, IPFIX Message integrity is important.

The IPFIX Messages themselves may also contain information of value to an attacker, including information about the configuration of the network as well as end-user traffic and payload data, so care must be taken to confine their visibility to authorized users. When an Information Element containing end-user payload information is exported, it SHOULD be transmitted to the Collecting Process using a means that secures its contents against eavesdropping. Suitable mechanisms include the use of either a direct point-to-point connection or the use of an encryption mechanism. It is the 
responsibility of the collecting Process to provide a satisfactory degree of security for this collected data, including, if necessary, anonymization of any reported data.

\subsection{Applicability of TLS and DTLS}

Transport Layer Security (TLS) [RFC4346] and Datagram Transport Layer Security (DTLS) [RFC4347] were designed to provide the confidentiality, integrity, and authentication assurances required by the IPFIX protocol, without the need for pre-shared keys.

With the mandatory SCTP and PR-SCTP transport protocols for IPFIX, DTLS [RFC4347] MUST be implemented. If UDP is selected as the IPFIX transport protocol, DTLS [RFC4347] MUST be implemented. If TCP is selected as the IPFIX transport protocol, TLS [RFC4346] MUST be implemented.

Note that DTLS is selected as the security mechanism for SCTP and PR-SCTP. Though TLS bindings to SCTP are defined in [RFC3436], they require all communication to be over reliable, bidirectional streams, and require one TLS connection per stream. This arrangement is not compatible with the rationale behind the choice of SCTP as an IPFIX transport protocol.

Note that using DTLS [RFC4347] has a vulnerability, i.e., a true man in the middle may attempt to take data out of an association and fool the sender into thinking that the data was actually received by the peer. In generic TLS for SCTP (and/or TCP), this is not possible. This means that the removal of a message may become hidden from the sender or receiver. Another vulnerability of using PR-SCTP with DTLS is that someone could inject SCTP control information to shut down the SCTP association, effectively generating a loss of IPFIX Messages if those are buffered outside of the SCTP association. In the future, techniques such as [dtls-for-sctp] could be used to overcome these vulnerabilities.

When using DTLS over SCTP, the Exporting Process MUST ensure that each IPFIX Message is sent over the same SCTP stream that would be used when sending the same IPFIX Message directly over SCTP. Note that DTLS may send its own control messages on stream 0 with full reliability; however, this will not interfere with the processing of stream 0 IPFIX Messages at the Collecting Process, because DTLS consumes its own control messages before passing IPFIX Messages up to the application layer. 
11.2. Usage

The IPFIX Exporting Process initiates the communication to the IPFIX Collecting Process, and acts as a TLS or DTLS client according to [RFC4346] and [RFC4347], while the IPFIX Collecting Process acts as a TLS or DTLS server. The DTLS client opens a secure connection on the SCTP port 4740 of the DTLS server if SCTP or PR-SCTP is selected as the transport protocol. The TLS client opens a secure connection on the TCP port 4740 of the TLS server if TCP is selected as the transport protocol. The DTLS client opens a secure connection on the UDP port 4740 of the DTLS server if UDP is selected as the transport protocol.

\subsection{Authentication}

IPFIX Exporting Processes and IPFIX Collecting Processes are identified by the fully qualified domain name of the interface on which IPFIX Messages are sent or received, for purposes of X.509 client and server certificates as in [RFC3280].

To prevent man-in-the-middle attacks from impostor Exporting or Collecting Processes, the acceptance of data from an unauthorized Exporting Process, or the export of data to an unauthorized Collecting Process, strong mutual authentication via asymmetric keys MUST be used for both TLS and DTLS. Each of the IPFIX Exporting and Collecting Processes MUST verify the identity of its peer against its authorized certificates, and MUST verify that the peer's certificate matches its fully qualified domain name, or, in the case of SCTP, the fully qualified domain name of one of its endpoints.

The fully qualified domain name used to identify an IPFIX Collecting Process or Exporting Process may be stored either in a subjectAltName extension of type dNSName, or in the most specific Common Name field of the Subject field of the X.509 certificate. If both are present, the subjectAltName extension is given preference.

Internationalized domain names (IDN) in either the subjectAltName extension of type dNSName or the most specific Common Name field of the subject field of the X.509 certificate MUST be encoded using Punycode [RFC3492] as described in section 4 of [RFC3490], "Conversion Operations".

\subsection{Protection against DoS Attacks}

An attacker may mount a denial-of-service (DoS) attack against an IPFIX collection system either directly, by sending large amounts of traffic to a collecting Process, or indirectly, by generating large amounts of traffic to be measured by a Metering Process. 
Direct denial-of-service attacks can also involve state exhaustion, whether at the transport layer (e.g., by creating a large number of pending connections), or within the IPFIX Collecting Process itself (e.g., by sending Flow Records pending Template or scope information, a large amount of Options Template Records, etc.).

SCTP mandates a cookie-exchange mechanism designed to defend against SCTP state exhaustion denial-of-service attacks. Similarly, TCP provides the "SYN cookie" mechanism to mitigate state exhaustion; SYN cookies SHOULD be used by any Collecting Process accepting TCP connections. DTLS also provides cookie exchange to protect against DTLS server state exhaustion.

The reader should note that there is no way to prevent fake IPFIX Message processing (and state creation) for UDP \& SCTP communication. The use of TLS and DTLS can obviously prevent the creation of fake states, but they are themselves prone to state exhaustion attacks. Therefore, Collector rate limiting SHOULD be used to protect TLS \& DTLS (like limiting the number of new TLS or DTLS session per second to a sensible number).

IPFIX state exhaustion attacks can be mitigated by limiting the rate at which new connections or associations will be opened by the Collecting Process, the rate at which IPFIX Messages will be accepted by the Collecting Process, and adaptively limiting the amount of state kept, particularly records waiting on Templates. These rate and state limits MAY be provided by a Collecting Process; if provided, the limits SHOULD be user configurable.

Additionally, an IPFIX Collecting Process can eliminate the risk of state exhaustion attacks from untrusted nodes by requiring TLS or DTLS mutual authentication, causing the collecting Process to accept IPFIX Messages only from trusted sources.

With respect to indirect denial of service, the behavior of IPFIX under overload conditions depends on the transport protocol in use. For IPFIX over TCP, TCP congestion control would cause the flow of IPFIX Messages to back off and eventually stall, blinding the IPFIX system. PR-SCTP improves upon this situation somewhat, as some IPFIX Messages would continue to be received by the collecting Process due to the avoidance of head-of-line blocking by SCTP's multiple streams and partial reliability features, possibly affording some visibility of the attack. The situation is similar with UDP, as some datagrams may continue to be received at the Collecting Process, effectively applying sampling to the IPFIX Message stream, implying that some forensics may be left. 
To minimize IPFIX Message loss under overload conditions, some mechanism for service differentiation could be used to prioritize IPFIX traffic over other traffic on the same link. Alternatively, IPFIX Messages can be transported over a dedicated network. In this case, care must be taken to ensure that the dedicated network can handle the expected peak IPFIX Message traffic.

11.5. When DTLS or TLS Is Not an Option

The use of DTLS or TLS might not be possible in some cases due to performance issues or other operational concerns.

Without TLS or DTLS mutual authentication, IPFIX Exporting Processes and Collecting Processes can fall back on using IP source addresses to authenticate their peers. A policy of allocating Exporting Process and Collecting Process IP addresses from specified address ranges, and using ingress filtering to prevent spoofing, can improve the usefulness of this approach. Again, completely segregating IPFIX traffic on a dedicated network, where possible, can improve security even further. In any case, the use of open collecting Processes (those that will accept IPFIX Messages from any Exporting Process regardless of IP address or identity) is discouraged.

Modern TCP and SCTP implementations are resistant to blind insertion attacks (see [RFC1948], [RFC4960]); however, UDP offers no such protection. For this reason, IPFIX Message traffic transported via UDP and not secured Via DTLS SHOULD be protected via segregation to a dedicated network.

\subsection{Logging an IPFIX Attack}

IPFIX Collecting Processes MUST detect potential IPFIX Message insertion or loss conditions by tracking the IPFIX sequence Number, and SHOULD provide a logging mechanism for reporting out-of-sequence messages. Note that an attacker may be able to exploit the handling of out-of-sequence messages at the Collecting Process, so care should be taken in handling these conditions. For example, a collecting Process that simply resets the expected Sequence Number upon receipt of a later Sequence Number could be temporarily blinded by deliberate injection of later sequence Numbers.

IPFIX Exporting and Collecting Processes SHOULD log any connection attempt that fails due to authentication failure, whether due to being presented an unauthorized or mismatched certificate during TLS or DTLS mutual authentication, or due to a connection attempt from an unauthorized IP address when TLS or DTLS is not in use. 
IPFIX Exporting and Collecting Processes SHOULD detect and log any SCTP association reset or TCP connection reset.

11.7. Securing the Collector

The security of the collector and its implementation is important to achieve overall security. However, it is outside the scope of this document.

\section{IANA Considerations}

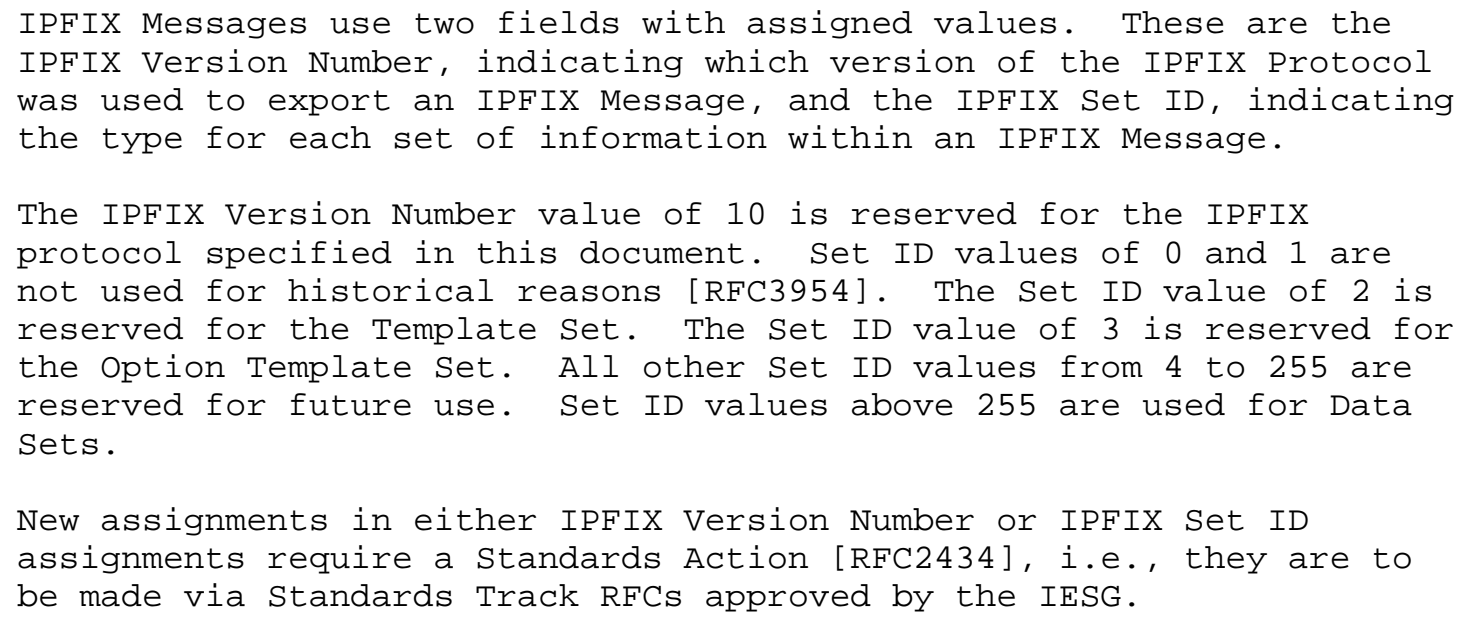




\section{Appendix A. IPFIX Encoding Examples}

This appendix, which is a not a normative reference, contains IPFIX encoding examples.

Let's consider the example of an IPFIX Message composed of a Template Set, a Data set (which contains three Data Records), an Options Template set and a Data set (which contains 2 Data Records related to the previous Options Template Record).

IPFIX Message:

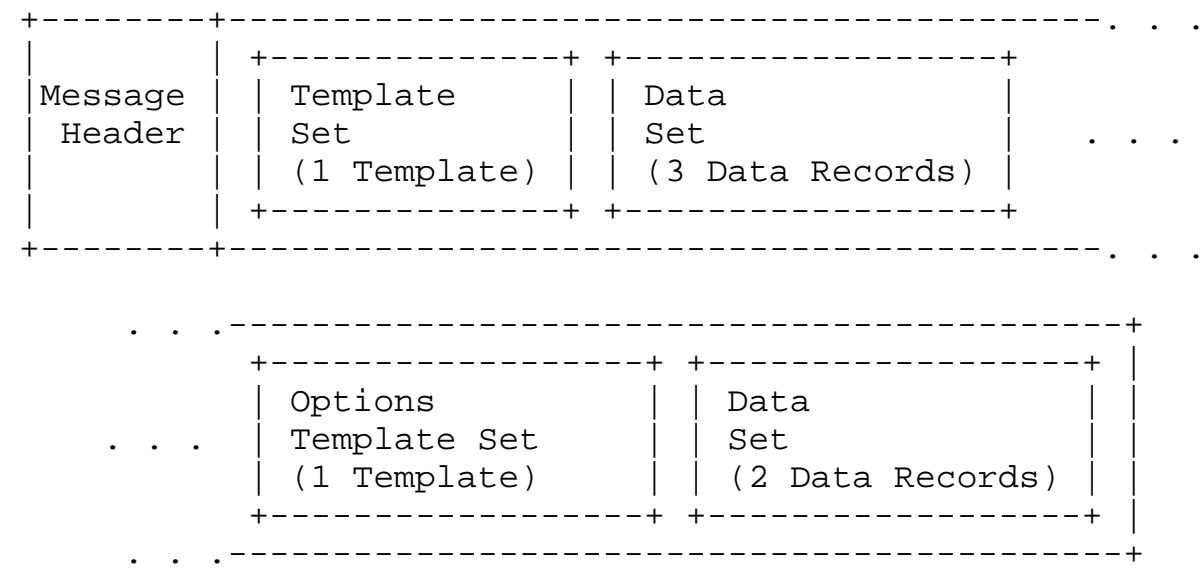

A.1. Message Header Example

The Message Header is composed of:

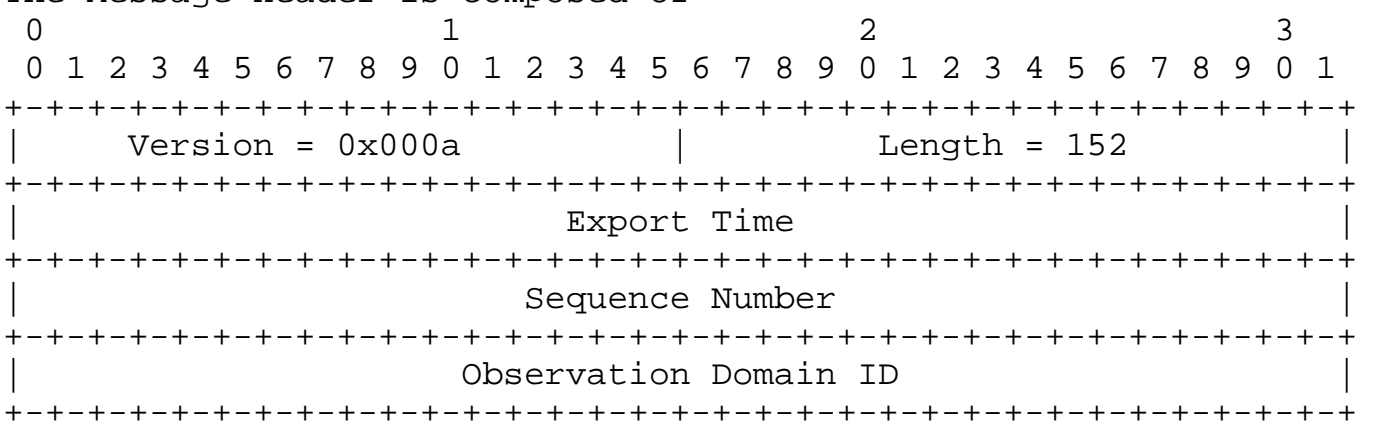




\section{A.2. Template set Examples}

\section{A.2.1. Template set Using IETF-Specified Information Elements}

We want to report the following Information Elements:

- The IPv4 source IP address: sourceIPv4Address in [RFC5102], with a length of 4 octets

- The IPv4 destination IP address: destinationIPV4Address in [RFC5102], with a length of 4 octets

- The next-hop IP address (IPv4): ipNextHopIPv4Address in [RFC5102], with a length of 4 octets

- The number of packets of the Flow: inPacketDeltacount in [RFC5102], with a length of 4 octets

- The number of octets of the Flow: inoctetDeltacount in [RFC5102], with a length of 4 octets

Therefore, the Template set will be composed of the following:

$\begin{array}{llllllllllllllllllllllllllllllllll}0 & 1 & 2 & 3 & 4 & 5 & 6 & 7 & 8 & 9 & 0 & 1 & 2 & 3 & 4 & 5 & 6 & 7 & 8 & 9 & 0 & 1 & 2 & 3 & 4 & 5 & 6 & 7 & 8 & 9 & 0 & 1\end{array}$
$+-+-+-+-+-+-+-+-+-+-+-+-+-+-+-+-+-+-+-+-+-+-+-+-+-+-+-+-+-+-+-+-+$

Set ID $=2$
Template ID 256

\section{A.2.2. Template Set Using Enterprise-Specific Information Elements}

We want to report the following Information Elements:

- The IPv4 source IP address: sourceIPv4Address in [RFC5102], with a length of 4 octets 
- The IPV4 destination IP address: destinationIPV4Address in [RFC5102], with a length of 4 octets

- An enterprise-specific Information Element representing proprietary information, with a type of 15 and a length of 4

- The number of packets of the Flow: inPacketDeltaCount in [RFC5102], with a length of 4 octets

- The number of octets of the Flow: inoctetDeltaCount in [RFC5102], with a length of 4 octets

Therefore, the Template set will be composed of the following:

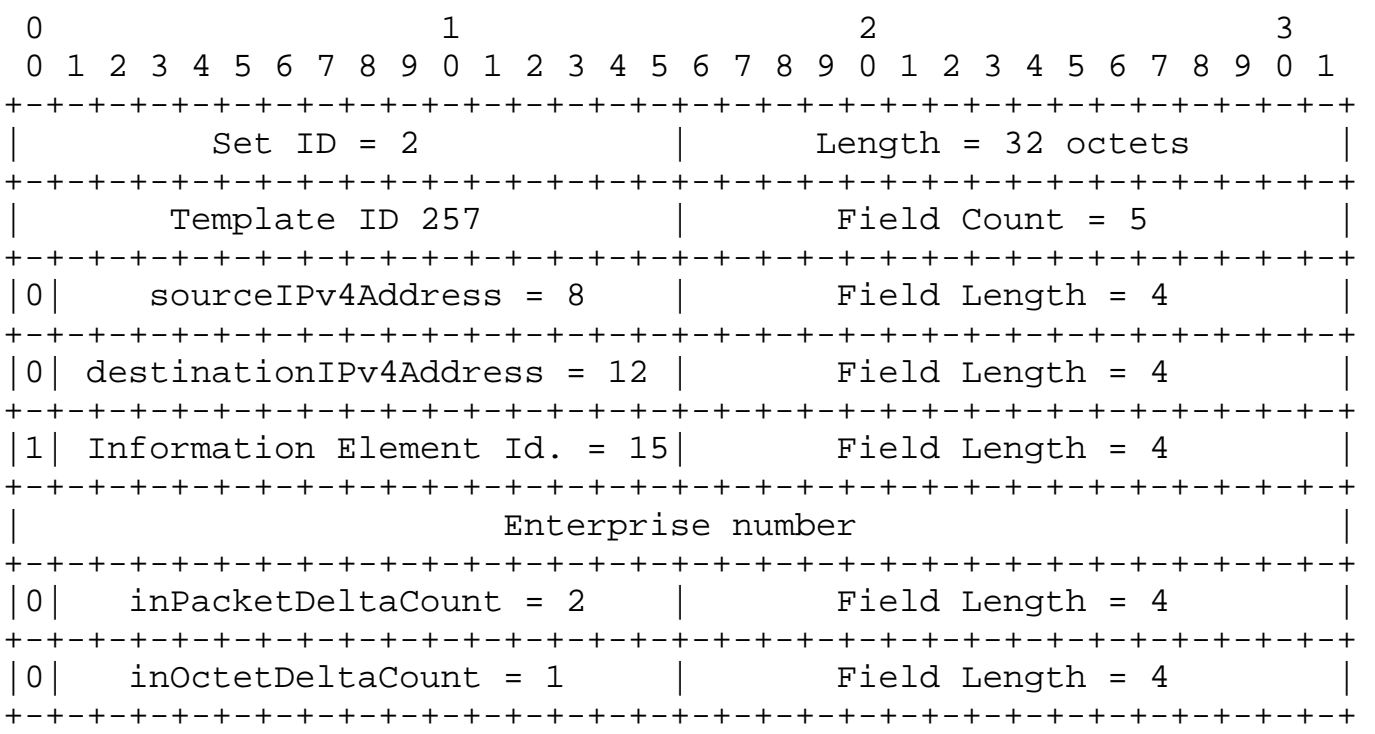




\section{A.3. Data set Example}

In this example, we report the following three flow Records:

\begin{tabular}{l|l|l|l|l} 
Src IP addr. & Dst IP addr. & Next Hop addr. & $\begin{array}{l}\text { Packet } \\
\text { Number }\end{array}$ & $\begin{array}{l}\text { Octets } \\
\text { Number }\end{array}$ \\
\hline----------1 & & &
\end{tabular}

$\begin{array}{llllllllll}0 & 1 & 2 & 3\end{array}$

$\begin{array}{llllllllllllllllllllllllllllllll}0 & 1 & 2 & 3 & 4 & 5 & 6 & 7 & 8 & 9 & 0 & 1 & 2 & 3 & 4 & 5 & 6 & 7 & 8 & 9 & 0 & 1 & 2 & 3 & 4 & 5 & 6 & 7 & 8 & 9 & 0 & 1\end{array}$ +-+-+-+-+-+-+-+-+-+-+-+-+-+-+-+-+-+-+-+-+-+-+-+-+-+-t-+-t-+-+-+-t

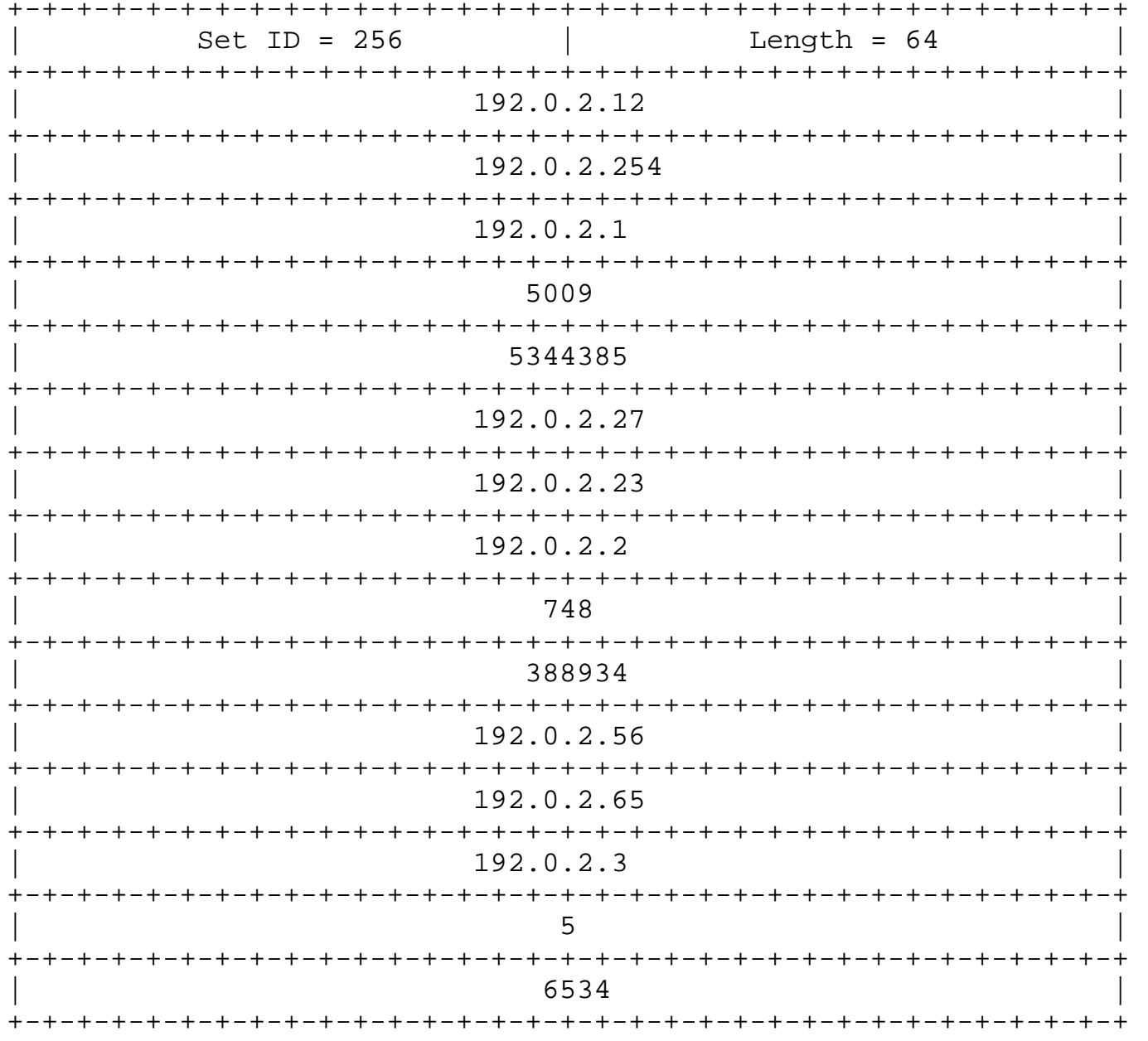

Note that padding is not necessary in this example. 


\section{A.4. Options Template Set Examples}

\section{A.4.1. Options Template Set Using IETF-Specified Information Elements}

Per line card (the router being composed of two line cards), we want to report the following Information Elements:

- Total number of IPFIX Messages: exportedPacketCount [RFC5102], with a length of 2 octets

- Total number of exported Flows: exportedFlowCount [RFC5102], with a length of 2 octets

The line card, which is represented by the linecardId Information Element [RFC5102], is used as the scope Field.

Therefore, the Options Template set will be:

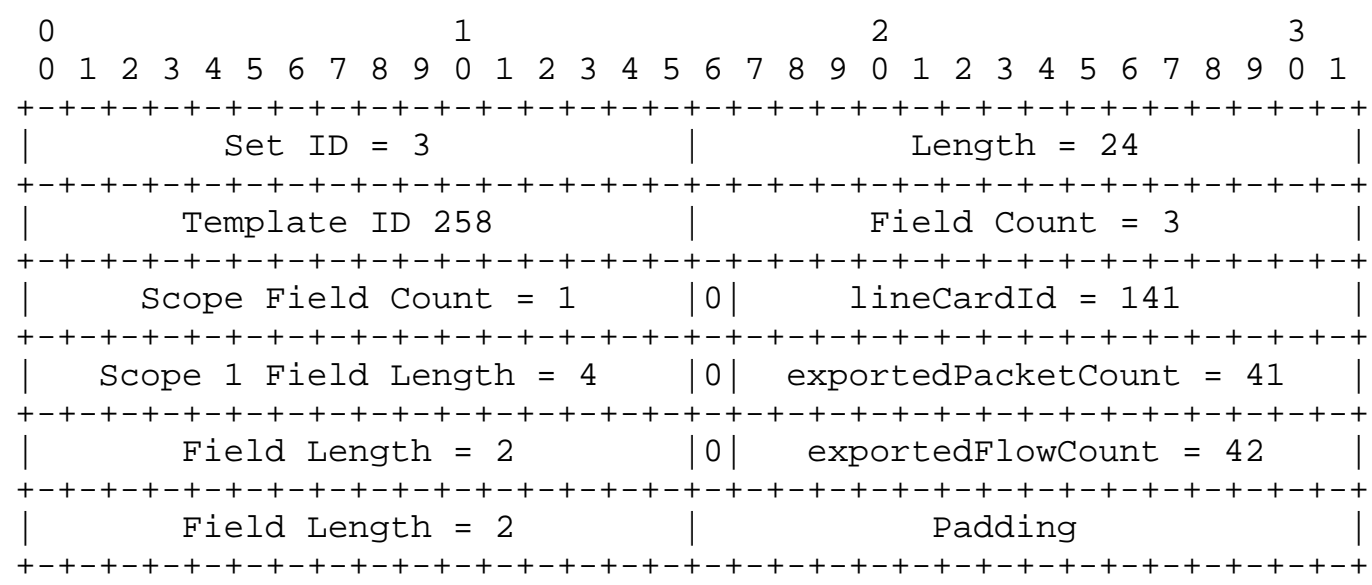

A.4.2. Options Template Set Using Enterprise-Specific Information Elements

Per line card (the router being composed of two line cards), we want to report the following Information Elements:

- Total number of IPFIX Messages: exportedPacketCount [RFC5102], with a length of 2 octets

- An enterprise-specific number of exported Flows, with a type of 42 and a length of 4 octets

The line card, which is represented by the linecardId Information Element [RFC5102], is used as the scope Field. 
The format of the Options Template set is as follows:

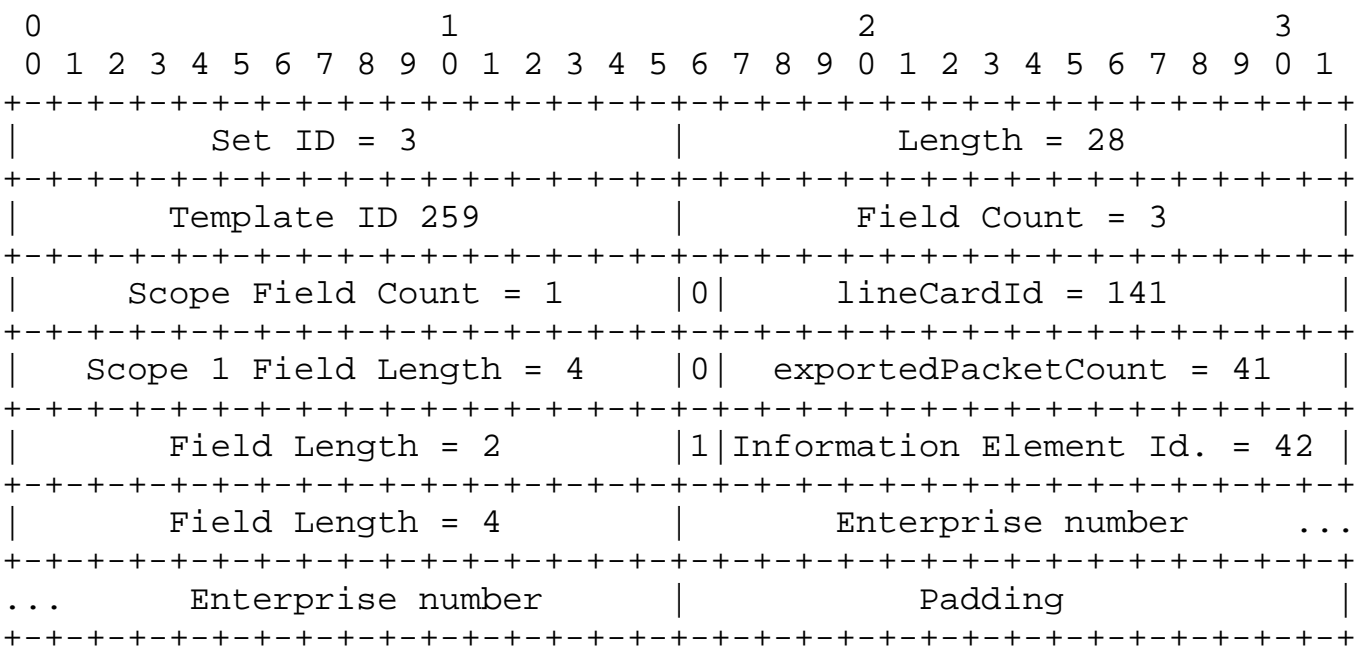

\section{A.4.3. Options Template Set Using an Enterprise-Specific Scope}

In this example, we want to export the same information as in the example in Section A.4.1:

- Total number of IPFIX Messages: exportedPacketCount [RFC5102], with a length of 2 octets

- Total number of exported Flows: exportedFlowCount [RFC5102], with a length of 2 octets

But this time, the information pertains to a proprietary scope, identified by enterprise-specific Information Element number 123. 
The format of the Options Template set is now as follows:

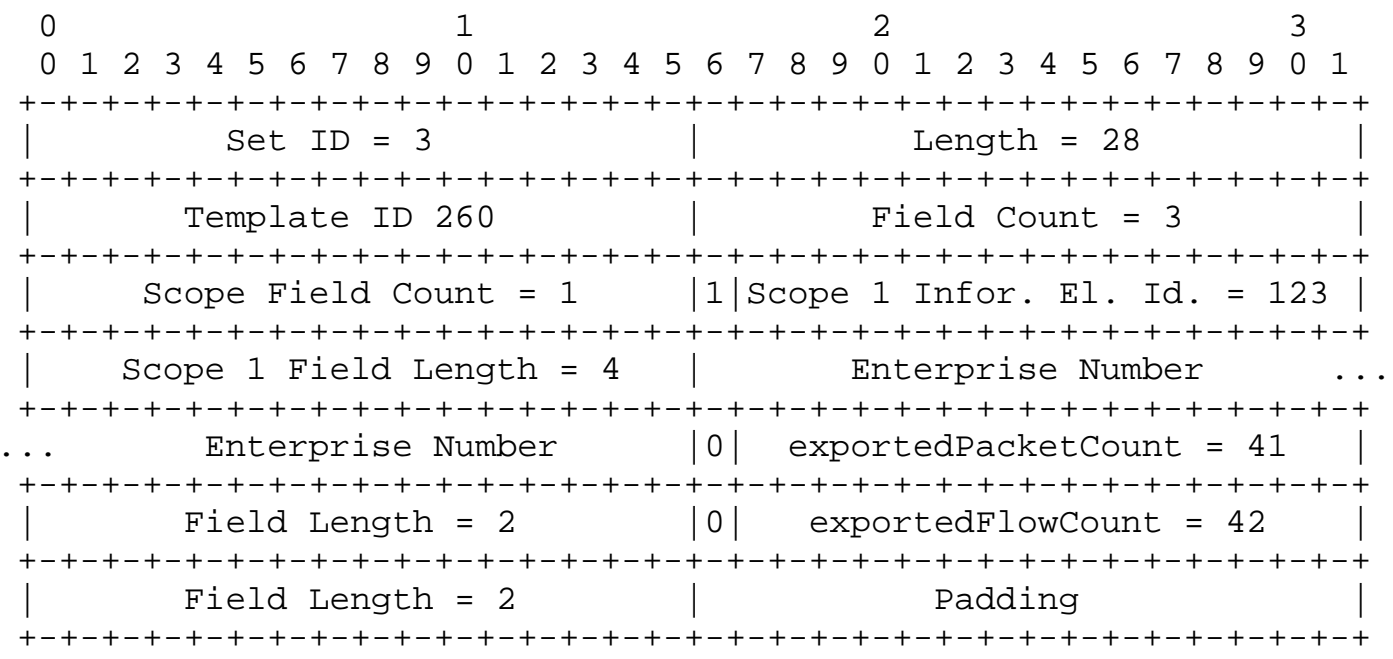

\section{A.4.4. Data Set Using an Enterprise-Specific Scope}

In this example, we report the following two Data Records:

\begin{tabular}{l|l|l} 
Line Card ID & IPFIX Message & Exported Flow Records \\
-------- & 10201 \\
Line Card 1 (lineCardId=1) & 345 & 20402 \\
Line Card 2 (lineCardId=2) & 690 &
\end{tabular}

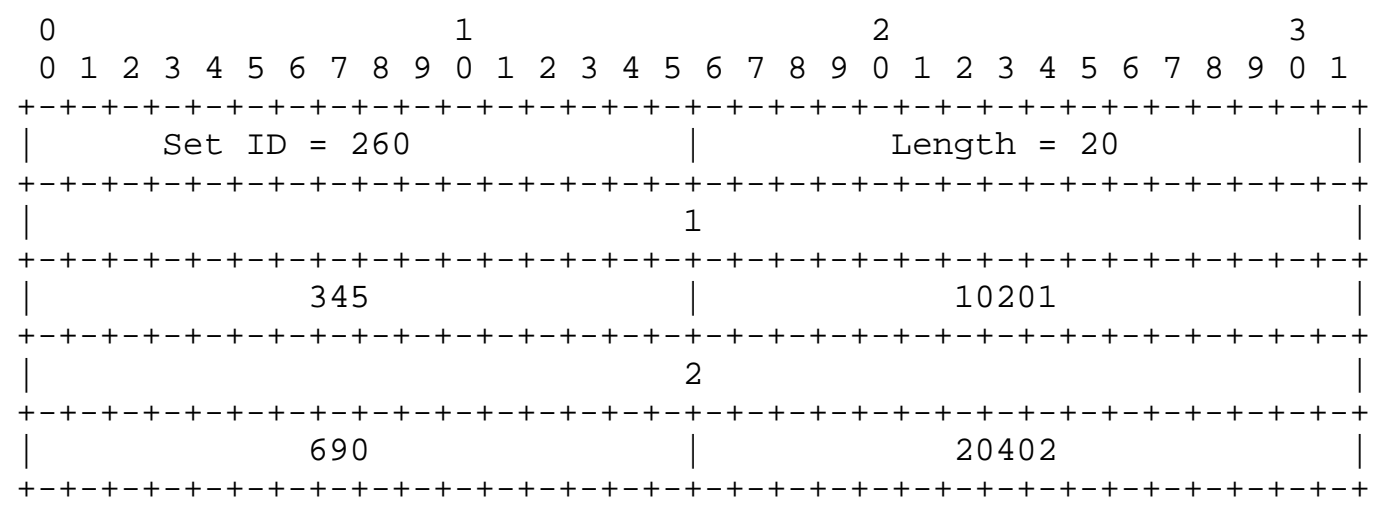




\section{A.5. Variable-Length Information Element Examples}

A.5.1. Example of Variable-Length Information Element with Length Inferior to 255 Octets

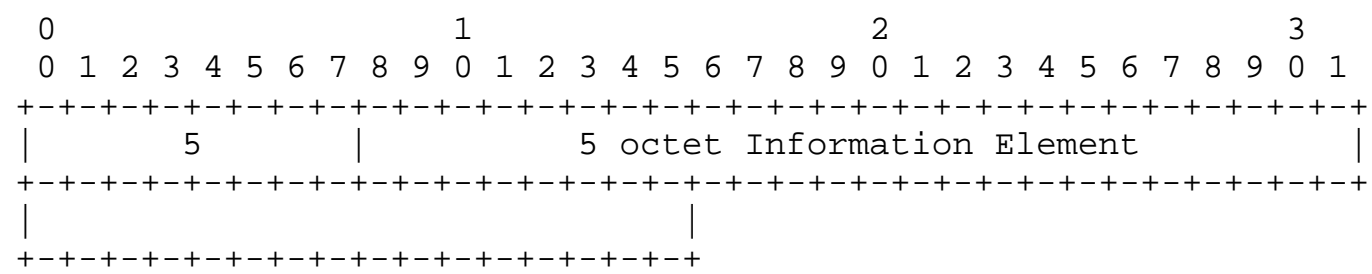

A.5.2. Example of Variable-Length Information Element with Length 255 to 65535 octets

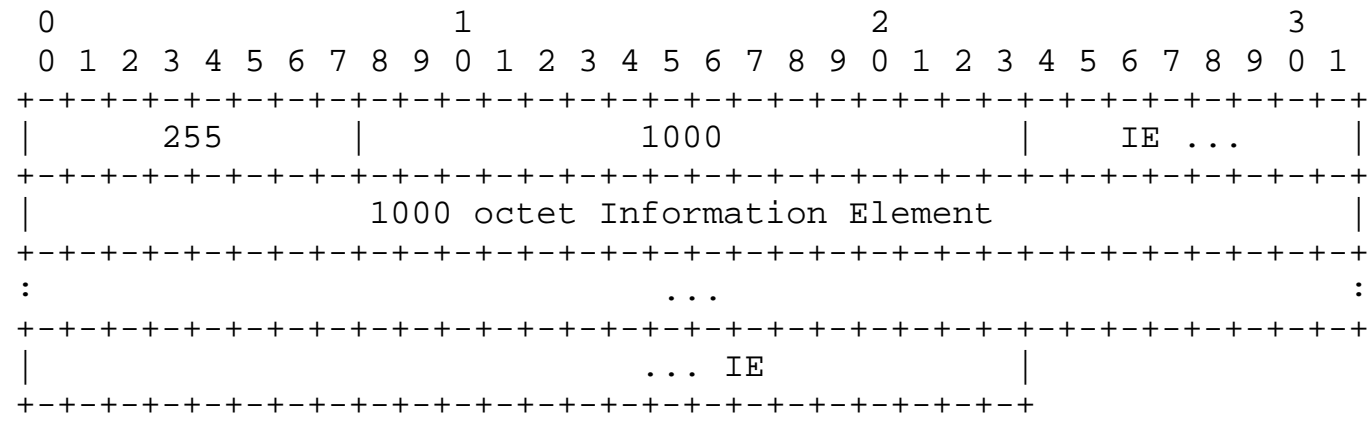

References

Normative References

[RFC1305] Mills, D., "Network Time Protocol (Version 3) Specification, Implementation and Analysis", RFC 1305, March 1992.

[RFC2119] Bradner, S., "Key words for use in RFCs to Indicate Requirement Levels", BCP 14, RFC 2119, March 1997.

[RFC2434]

Narten, T. and H. Alvestrand, "Guidelines for Writing an IANA Considerations section in RFCs", BCP 26, RFC 2434, October 1998 .

[RFC3280] Housley, R., Polk, W., Ford, W., and D. Solo, "Internet X.509 Public Key Infrastructure Certificate and Certificate Revocation List (CRL) Profile", RFC 3280, April 2002 . 
[RFC3436] Jungmaier, A., Rescorla, E., and M. Tuexen,

"Transport Layer Security over Stream Control

Transmission Protocol", RFC 3436, December 2002.

[RFC3758] Stewart, R., Ramalho, M., Xie, Q., Tuexen, M., and P. Conrad, "Stream Control Transmission Protocol (SCTP) Partial Reliability Extension", RFC 3758, May 2004.

[RFC4346] Dierks, T. and E. Rescorla, "The Transport Layer Security (TLS) Protocol Version 1.1", RFC 4346, April 2006 .

[RFC4347] Rescorla, E. and N. Modadugu, "Datagram Transport Layer Security", RFC 4347, April 2006.

[RFC3490] Faltstrom, P., Hoffman, P., and A. Costello, "Internationalizing Domain Names in Applications (IDNA)", RFC 3490, March 2003.

[RFC3492] Costello, A., "Punycode: A Bootstring encoding of Unicode for Internationalized Domain Names in Applications (IDNA)", RFC 3492, March 2003.

[RFC4960] Stewart, R., Ed., "Stream Control Transmission Protocol", RFC 4960, September 2007.

[RFC5102] Quittek, J., Bryant S., Claise, B., Aitken, P., and J. Meyer, "Information Model for IP Flow Information Export", RFC 5102, January 2008.

$[\mathrm{TCP}]$

Postel, J., "Transmission Control Protocol", STD 7, RFC 793, September 1981.

[UDP] Postel, J., "User Datagram Protocol", STD 6, RFC 768, August 1980 .

Informative References

[IPFIX-ARCH] Sadasivan, G., Brownlee, N., Claise, B., and J. Quittek, "Architecture Model for IP Flow Information Export", Work in Progress, September 2006.

[IPFIX-AS] Zseby, T., Boschi, E., Brownlee, N., and B. Claise, "IPFIX Applicability", Work in Progress, June 2007.

[PEN] IANA Private Enterprise Numbers registry http: //www.iana.org/assignments/enterprise-numbers . 
[RFC1948] Bellovin, S., "Defending Against Sequence Number Attacks", RFC 1948, May 1996.

[RFC2579] McCloghrie, K., Perkins, D., and J. Schoenwaelder, "Textual Conventions for SMIv2", STD 58, RFC 2579, April 1999.

[RFC3917] Quittek, J., Zseby, T., Claise, B., and S. Zander, "Requirements for IP Flow Information Export

(IPFIX) ", RFC 3917, October 2004.

[RFC3550] Schulzrinne, H., Casner, S., Frederick, R., and V. Jacobson, "RTP: A Transport Protocol for Real-Time Applications", STD 64, RFC 3550, July 2003.

[RFC3954] Claise, B., Ed., "Cisco Systems NetFlow Services Export Version 9", RFC 3954, October 2004.

[IEEE.754.1985] Institute of Electrical and Electronics Engineers, "Standard for Binary Floating-Point Arithmetic", IEEE Standard 754, August 1985 .

[dtls-for-sctp] Tuexen, M. and E. Rescola, "Datagram Transport Layer Security for Stream Control Transmission Protocol", Work in Progress, November 2007.

Acknowledgments

We would like to thank the following persons: Ganesh Sadasivan for his significant contribution during the initial phases of the protocol specification; Juergen Quittek for the coordination job within IPFIX and PSAMP; Nevil Brownlee, Dave Plonka, Paul Aitken, and Andrew Johnson for the thorough reviews; Randall stewart and Peter Lei for their SCTP expertise and contributions; Martin Djernaes for the first essay on the SCTP section; Michael Behringer and Eric Vyncke for their advice and knowledge in security; Michael Tuexen for his help regarding the DTLS section; Elisa Boschi for her contribution regarding the improvement of SCTP sections; Mark Fullmer, Sebastian Zander, Jeff Meyer, Maurizio Molina, Carter Bullard, Tal Givoly, Lutz Mark, David Moore, Robert Lowe, Paul Calato, and many more, for the technical reviews and feedback. 


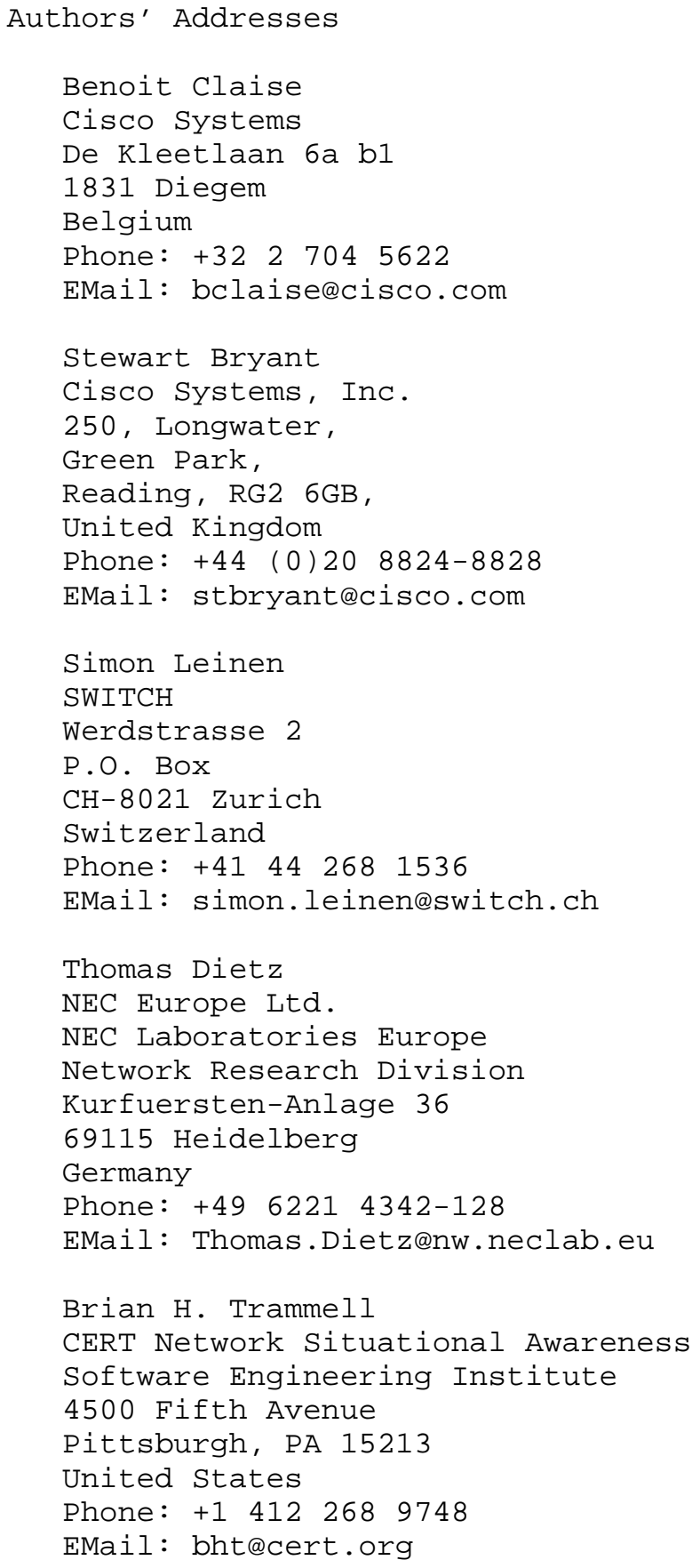


Full Copyright statement

Copyright (C) The IETF Trust (2008).

This document is subject to the rights, licenses and restrictions contained in BCP 78, and except as set forth therein, the authors retain all their rights.

This document and the information contained herein are provided on an "AS IS" basis and THE CONTRIBUTOR, THE ORGANIZATION HE/SHE REPRESENTS OR IS SPONSORED BY (IF ANY), THE INTERNET SOCIETY, THE IETF TRUST AND THE INTERNET ENGINEERING TASK FORCE DISCLAIM ALL WARRANTIES, EXPRESS OR IMPLIED, INCLUDING BUT NOT LIMITED TO ANY WARRANTY THAT THE USE OF THE INFORMATION HEREIN WILL NOT INFRINGE ANY RIGHTS OR ANY IMPLIED WARRANTIES OF MERCHANTABILITY OR FITNESS FOR A PARTICULAR PURPOSE.

Intellectual Property

The IETF takes no position regarding the validity or scope of any Intellectual Property Rights or other rights that might be claimed to pertain to the implementation or use of the technology described in this document or the extent to which any license under such rights might or might not be available; nor does it represent that it has made any independent effort to identify any such rights. Information on the procedures with respect to rights in RFC documents can be found in BCP 78 and BCP 79 .

Copies of IPR disclosures made to the IETF Secretariat and any assurances of licenses to be made available, or the result of an attempt made to obtain a general license or permission for the use of such proprietary rights by implementers or users of this specification can be obtained from the IETF on-line IPR repository at http://www.ietf.org/ipr.

The IETF invites any interested party to bring to its attention any copyrights, patents or patent applications, or other proprietary rights that may cover technology that may be required to implement this standard. Please address the information to the IETF at ietf-ipreietf.org. 